\title{
Notch Signalling in context
}

\author{
Sarah J Bray \\ Physiology Development and Neuroscience, \\ University of Cambridge \\ Downing Street, \\ Cambridge, CB2 3DY, UK \\ +44-1223-765222 \\ sjb32@cam.ac.uk
}




\section{Author Information}

Sarah Bray is Professor of Developmental Biology at the University of Cambridge. Her career began with studies of translational regulation in sea urchin embryos (with Tim Hunt), for her PhD. From there she moved to Harvard for her postdoctoral research, working with two groups (Jay Hirsh and Fotis Kafatos) that were harnessing transgenesis to study gene regulation. There she discovered the first member of the Grainy head family of transcription factors. Her research into the Notch pathway began when she returned to University of Cambridge to set up her own group. Their early work investigating Notch pathway functions in Drosophila began to highlight the diverse roles that the pathway plays in development. Since then her group have continued to investigate the basis for this diversity, with an emphasis on the mechanisms at the transcriptional heart of the pathway.

\section{Key Points:}

- The highly conserved Notch cell-cell signalling pathway operates in many different contexts where the consequences can differ widely, despite the fact that the core pathway is very simple.

- Many different types of regulation contribute to the differing outcomes, ranging from tissue level co-ordination to nuclear governance.

- The pattern of expression of the ligands, receptors and critical modifying enzymes is one level of regulation that is common to many signalling pathways. However, the one-to-one interaction between ligand and receptor places extra emphasis on this, especially as they can cis-inhibit one another when present in the same cells.

- 'Topological' tissue organization and the extent of cell-cell contacts is likely to be of unusual significance in influencing the levels of Notch activation, because the ligands are trans-membrane proteins.

- Nuclear context, in the form of cell-type specific transcription factors and chromatin organization is a primary level of control in generating qualitatively different outcomes from Notch activation. In addition, the wiring of the regulatory network within the signal receiving cells contributes to the diversity of responses and to the nature of its cross-talk with other signalling pathways.

- Together the mechanisms make the Notch pathway versatile and able to undertake many different roles. But they are also susceptible to perturbations, and may be a contributory factor in Notch-related diseases. 


\begin{abstract}
Since it became evident that the highly conserved Notch signalling pathway functions in many different developmental and homeostatic processes, questions have arisen about how this pathway can achieve such diverse outcomes. With a direct route from the membrane to the nucleus, the Notch pathway has fewer opportunities for regulation than many other signalling pathways, yet it generates exquisitely patterned structures. More confusingly, its activity promotes growth in some circumstances but cell death in others. This review will consider the regulatory mechanisms that shape the activity of the Notch pathway and its outcome, enabling it to generate biological consequences that are appropriate for each context.
\end{abstract}

\title{
Introduction:
}

Despite the fact that the core Notch pathway operates in vastly different developmental and disease contexts, from stem cell regulation and heart morphogenesis to cancers and cardiomyopathies, it is relatively simple in its operation. Ligand-mediated activation induces a series of proteolytic cleavages in members of the Notch family of receptors, which release the Notch intracellular domain, NICD;. (Figure 1a and Box 1). Once released, the NICD enters the nucleus and, together with the DNA-binding protein CSL (CBF1/Suppressor of Hairless/Lag1) and the co-activator Mastermind (Mam), stimulates transcription of target genes (Figure 1a) ${ }^{1-4}$. Thus, no amplification of the signal can occur, unlike in many other pathways, and no intermediates are present between the membrane and the nucleus -NICD takes responsibility for implementing pathway activation.

Given this relative simplicity - receptor-ligand interactions release the bioactive NICD - how can the canonical Notch pathway coordinate so many diverse biological outcomes? One Notch receptor and two ligands - Delta and Serrate (Ser) - exist in Drosophila melanogaster while vertebrates have four Notch paralogues (Notch1-4) and a similar diversification of ligands (referred to as Delta-like ligands (DLLs) and Jagged in mammals). This repertoire clearly cannot account for the diversity of Notch pathway signalling outcomes, indicating that other mechanisms of regulation exist. In this Review, we will illustrate features that enable the Notch pathway to function differently according to its setting and that help to explain the myriad of roles that the pathway has in development and disease. It is only possible to draw on a small subset of examples owing to space limitations, but these examples highlight fundamental principles that should be widely applicable across the different contexts where the Notch pathway operates.

\section{Ligand-receptor landscapes [L1 Heading]}

Early models suggested that Notch-mediated developmental patterning occurred when stochastic differences in ligand-receptor interactions directed signalling within fields of cells with near uniform levels of ligand expression. It now seems that this scenario is the exception rather than the rule: the expression profiles of ligands, receptors and several modifying enzymes have important roles in defining the outcome. Furthermore, other signalling pathways can regulate these expression patterns to augment, inhibit or modulate Notch pathway activity, providing an important mechanism of crosstalk.

\section{Expression of canonical ligands and receptors [L2 heading]}

The classic paradigm of lateral inhibition, which is a common phenomenon during precursor patterning, assumed that the driving force for signalling arose through stochastic differences in ligand levels. The initial bias would be reinforced by a negative-feedback loop so that ligand expression became repressed in signalreceiving cells, generating a spaced distribution of signalling cells and receiving cells that explained the distribution of precursors ${ }^{5}$. It now seems that signalling rarely 
relies on uniform ligands; rather, their spatial and temporal regulation directs Notch signalling profiles in many contexts and is an important mode for regulation by other signalling pathways. Although much regulation of ligand expression occurs at the transcriptional level, micro-RNAs (miRs) also contribute. For example, miR8/miR200 targets the 3'UTRs of Ser and Jagged1 (Jag1) to fine-tune the levels of protein produced in $D$. melanogaster and in human tissues, respectively ${ }^{6,7}$.

One example of how ligand expression dictates the spatial pattern of signalling occurs during the formation of the growth organizer in $D$. melanogaster wing imaginal discs. Here, Ser is produced exclusively in the dorsal territory owing to its regulation by the spatially restricted Apterous transcription factor ${ }^{8,9}$, and specifically generates a stripe of Notch activity in adjacent ventral cells to create a boundary that organizes growth of the tissue ${ }^{8}$. Dynamic changes in expression of both ligand and receptor also help to drive oscillations in Notch signaling ${ }^{10}$ such as occur during somitogenesis in the presomitic mesoderm. Here, Notch1 expression is dependent on its' pathway activity which might help to reinforce signalling, whereas ligand expression is Wnt regulated, thus ensuring that the pattern of Notch activity is instructed by another main component involved in the somite clock $^{11,12}$.

In addition, interplay between different ligands is frequently required to set the correct balance of precursor cells in a number of situations. For example, in the ear, a broad 'inductive' signal by Ser/ Jag1 confers the neurogenic potential of the placode ${ }^{13} 14$ before subsequent dispersed expression of DLLs in emerging precursors inhibits the surrounding cells to ensure a single precursor is generated at each position ${ }^{15-18}$. Likewise, to establish the branching pattern of blood vessels the production of DLL4 in tip cells prevents neighbouring cells from adopting the same fate ${ }^{19,20}$, whereas Jag1 acts as a potent proangiogenic regulator that antagonizes DLL4-Notch signalling to favour new sprouting.

Although the fundamental consequences of ligand binding are the same -- cleavage of Notch receptors to produce NICD -- they nevertheless often elicit different outcomes $^{21}$. One possibility is that different ligands bring about different strengths (or durations) of intracellular signal. For example, Jag1 and DLL1 have lower measured affinities for Notch1 than does DLL4, possibly owing to differences in the orientation of the amino -terminal and Delta/Serrate/Lag-2 (DSL) domains involved in the binding interface ${ }^{22}$. Indeed, in the haemangiogenic endothelium, specification of haematopoietic stem cells involves a low-strength Jag1-dependent signal whereas specification of endothelial arterial cells requires a high-strength DII4 signal ${ }^{23}$. Strikingly, cells that received the low-strength Jag1-induced Notch activity appeared unresponsive to a high-strength DLL4 signal. Although it is unclear how this lack of response arises, if the expression of Jag1 precedes that of DLL4, it could switch on an inhibitory programme or cause cell re-arrangements to disrupt contacts with DLL4. Similarly, in the inner ear Jag1 might elicit a lower level of Notch1 activation than DLL1 does, as Jag1 can induce the expression of Hey1, which requires a low threshold, but not Hes5, which requires a higher level of signalling ${ }^{24}$.

In animals with multiple Notch paralogues, the deployment of different receptors will also influence signalling outcomes. In humans, mutations in different paralogues have different disease consequences (for example, only defects in Notch3 cause CADASIL (cerebral autosomal-dominant arteriopathy with subcortical infarcts and leukoencephalopathy), emphasizing different receptor roles ${ }^{21,25,26}$. Although these differences might be partly explained by variations in their expression patterns, examples exist where individual paralogues - for example, Notch1 and Notch2 make different contributions even when expressed in identical patterns. Indeed, Notch1 and Notch2 were found to have opposite effects on the growth of one specific 
tumor cell-type ${ }^{27}$. However, when the intracellular domains of Notch1 and Notch2 were swapped within each normal gene context their intrinsic activities were similar ${ }^{28,}{ }^{29}$ but, in some contexts, the amount or stability of the NICD moieties differed ${ }^{29}$. This may reflect differences in their potential for post-translational modifications. These observations emphasize the possibility that, ultimately, the amount or duration of the NICD 'signal' produced will depend on the specific ligand-receptor pairs that are engaged, as well as the availability of the metalloproteases essential for the activating cleavages. Whether such NICD differences would result in quantitative or in qualitative differences in the downstream signalling output is an important question that remains to be resolved.

\section{Contributions from cis inhibition [L2 heading]}

Notch signalling is also highly sensitive to the relative levels of ligands and receptors owing to cis inhibition, an inhibitory interaction that occurs when ligand and receptor are present in the same cell (Figure 2) ${ }^{30}$. First identified through genetic experiments in $D$. melanogaster ${ }^{31,32}$, the precise mechanism for cis inhibition remains elusive, although some studies suggest that Notch molecules are targeted for degradation once they have undergone ligand interaction ${ }^{33} 34$.

Modelling experiments suggest that an ultrasensitive switch between two mutually exclusive cell states: signal-sending and signal-receiving could be generated by Notch having a sharp ligand threshold for cis inhibition combined with a graded response to trans-acting ligand ${ }^{35}$. One example of a cis-inhibitory interaction affecting the signalling outcome occurs during photoreceptor specification in $D$. melanogaster. here, loss of DI-mediated cis inhibition reversed the direction of lateral signalling, thereby generating the wrong complement of photoreceptors ${ }^{36}$. Likewise, cis inhibition helps to stabilize tip and stalk fates during angiogenesis, and hence prevents hybrid tip-stalk cells forming ${ }^{37}$. In addition, different Notch receptors might also cis inhibit one another, adding another potential mechanism to fine-tune signal reception ${ }^{38}$. The balance between cis-interactions and trans-interactions is thus likely to be important in signalling outcomes (Figure 2a).

Intriguingly, the mammalian ligand DLL3 might operate only in a cis-inhibitory mode. In cell-based assays, DLL3 was unable to activate signalling in trans but, when coexpressed with Notch1, it could prevent Notch1responding to other ligands on neighbouring cells ${ }^{39}$. In vivo, the loss of DLL3 led to increased Notch activity during T-cell development ${ }^{40}$ and to defects in Notch1 signalling in the presomitic mesoderm ${ }^{41}$. It is not fully clear why DLL3 might have a uniquely inhibitory role, although its highly divergent DSL domain might be a contributory factor; however, by doing so, it adds another strand of pathway regulation in vertebrates.

\section{Deployment of Fringes and other modifying enzymes [L2 heading]}

Further influencing Notch signalling is the presence of enzymes that modify the extracellular domains (ECDs) of ligands and receptors and modulate their ability to signal (Figure 2b). Although modifications to ligands might be important, most focus has been on receptors, the activity of which is profoundly affected by glycosylation of EGF repeats in the ECD. O-fucosyltransferases, which add fucose to serine and threonine residues, and O-glucosyltransferases, which add glucose to serines, are essential for optimal Notch signalling ${ }^{42,43}$. Many O-fucose monosaccharides on Notch can subsequently be extended (with $\mathrm{N}$-acetylglucosamine) by the Fringe proteins, with differing effects on Notch, depending on the sites modified and the ligands present ${ }^{42}$.

Fringe-mediated modifications could influence specific ligand-receptor interactions. Structural studies indicate that elongation of an O-fucose on EGF repeat 12 of Notch 
receptors could provide additional energetic contributions to Notch-ligand interfaces 22. This modification enhanced binding of DLL1 and Jag1 to a greater extent than binding to DLL4, possibly because the inherent affinity of DLL4 is relatively high even in the absence of glycosylation ${ }^{22}$. Whether or not the enhanced ligand binding translates into increased pathway activity is uncertain. In some cases, where the affinity of Jag1 for the receptor was increased by the presence of Fringe proteins, the effects on transcriptional output were reduced ${ }^{44}$. Concomitant changes to cis inhibition, to competition with other ligands or to other sites in the receptor are possible explanations for this apparent anomaly.

Fringe proteins are thus likely to modulate the ability of cells to send and receive signals in a manner that is highly dependent on the cocktail of ligands (and Fringe proteins) present (Figure 2b). For example, in D. melanogaster wing discs, modifications by Fringe render Notch insensitive to Ser ${ }^{45}$. Here, co-expression of Ser and Fringe in dorsal cells guarantees that Ser can only signal to the adjacent ventral territory ${ }^{8}$. At the same time, DI is enriched in ventral cells and its binding is enhanced by Fringe modifications ${ }^{45,46}$. The combined effects generate a stripe of cells with Notch activity that straddles the boundary. Likewise, when high levels of both Jag1 and Fringe are present in stalk cells, Fringe is thought to make Jag1 into an effective competitor for the more signalling proficient DLL4, which prevents DLL4 from signalling between adjacent stalk cells, and thereby inhibits excessive sprouting ${ }^{47}$. Similarly, during ventricular development, temporal modulation of Mfng enables sequential Notch activation to drive different morphological processes; MFng and Dll4 downregulation in the endocardium allows these cells to respond to myocardial Jag1/ Jag2 and generate a functional ventricular wall ${ }^{48}$. Finally, in the spinal cord, where Fringes enhance DLL-activated Notch signalling and block that of Jag1, the consequence of their patterned expression (controlled by homeodomain proteins) is domain-wide Notch activation by either DII or Jag1, and a suppression of signalling across progenitor domain boundaries ${ }^{49}$.

Experiments modelling the outcome of Fringe modifications have illustrated how these could impact on the relative signalling capabilities of cells. For example, cells expressing Lunatic Fringe (Lfng) or Manic Fringe (Mfng) in combination with Jag1 and Notch 1 acquired the ability to send and receive signals simultaneously, but only using different ligands ${ }^{50}$. This observation could be explained if Fringe modifications weaken cis interactions between Jag1 and Notch1, consequently making high surface levels of both available, and also prevent Notch1 from being trans activated by Jag1 (Figure 2b). Notch1 could thus only receive signals from DLL on adjacent cells but Jag1 would itself be free to signal to neighbouring cells. Such a model fits well with the observations at the dorsal-ventral boundary of the $D$. melanogaster wing and with the tip/stalk decision in angiogenesis, and highlights the profound effect of the patterned deployment of these molecules on signalling outcomes.

\section{Topological context [L1 heading]}

Notch receptors and their ligands are transmembrane proteins. Furthermore, endocytosis is known to be required for ligand activity ${ }^{51,52}$. Mechanisms that transport the proteins to and from the correct places in the cell are therefore likely to have an important impact on signalling. Although it has largely been assumed that ligand-receptor interactions occur at sites where cells are tightly opposed, several observations are challenging this assumption, and highlight that the dimensions and stability of the contact area might have important roles.

\section{Endocytic trafficking and ubiquitin ligases [L2 heading]}


Factors that modulate endocytosis and trafficking of receptors and ligands have a number of important consequences on pathway activity ${ }^{53,54}$. First, endocytosis of the ligand after it has engaged with its Notch receptor is thought to generate the force on the receptor that exposes the protease cleavage site within the negative regulatory (NRR) 'cage' ${ }^{55} 56,57$.(Figure 1b). In the absence of certain E3 ubiquitin ligases of the Mindbomb or Neuralized families, ligand endocytosis is prevented with concomitant loss of signalling ${ }^{58,5952,60-6364}$. As ubiquitylation of the ligand intracellular domains by these enzymes is critical for ligand activity, the presence of Mib and/or Neur determines which cells can send a signal. During asymmetric division, for example, directional Notch signalling is achieved, in part, by the polarized segregation of these E3 ligases into one of two daughter cells, depending on the underlying cell polarity ${ }^{65,66} 67$.

Second, receptor trafficking affects pathway activity not only by determining receptor levels on the cell surface but also because ligand-independent activation can occur during this process ${ }^{53,54,68}$. Normally, while some of the endocytosed Notch is likely recycled to the membrane, a large fraction is targeted for degradation. When this fraction of the receptor fails to be properly routed into the inner luminal vesicles of maturing lysosomes - for example, in response to mutations that affect the ESCRT complex ${ }^{69-72}$ this can result in ligand-independent Notch activation, although the precise mechanisms that underly this phenomenon are unknown. Most likely, the conditions encountered in the lysosome promote ligand-independent activation by destabilizing the NRR. Proteins that regulate endolysosomal transport have a concomitant effect on Notch trafficking that can, in some cases, result in receptor activation in normal physiological conditions. These include the E3 ligases Deltex and Itch/Suppressor of Deltex, which, by ubiquitylating Notch, alter both the receptor levels on the membrane and the amount of ligand-independent activation ${ }^{73-75}$

Numb is another factor that regulates cell-fate decisions via its powerful effects on receptor trafficking; its depletion in several lineages results in ectopic Notch activity. During sensory organ development, Numb is differentially segregated into one of two daughter cells, in which it inhibits Notch activity to bring about specific 'Notch off' fates ${ }^{76-80}$. Numb achieves this inhibition either by promoting Notch internalization or by altering the route taken after Notch is endocytosed ${ }^{81,82}$. Notably, this phenomenon only occurs in certain contexts, despite Numb being present more widely. In $D$. melanogaster, this specificity depends on the adaptor Sanpodo, which couples Numb localization with Notch trafficking ${ }^{82-84}$. Adaptors analogous to Sanpodo are likely to perform a similar task in other species.

Third, spatial regulation of trafficking also has the potential to affect the geometry of signalling. For example, endocytic depletion of Notch from the cleavage furrow after the division of sensory organ precursor cells in $D$. melanogaster is important to enable unidirectional signalling in the progeny ${ }^{85}$. The asymmetrical distribution of endosomes containing receptors and/or ligands is also associated with a bias in signalling to promote specific cell fates in neural lineages ${ }^{86,87}$. In addition, by affecting the activity and/or localization of the $\gamma$-secretase complex, which is required for the release of the NICD, proteins that organize cellular polarity (for example, Crumbs $^{88}$ and hibris ${ }^{89}$ ) can consequently affect Notch signalling. Finally, beyond regulating the activity of the ligands and receptor, endocytic trafficking might also be important for localizing functional pools of ligand and receptor to the appropriate cellular subdomains, although many questions remain about where these subdomains are located. 
Because Notch signalling occurs between cells that are in contact with one another, the organization of the tissue is likely to influence the levels or patterns of signalling, such that the strength and periodicity of signalling could depend on the extent or durability of the adhesive contacts between cells. For example, models suggest that, under conditions in which the extent of cell-cell contact is greater than the diffusion range of the ligand within the membrane, the signal generated will be proportional to the contact area ${ }^{90}$. Signalling might also be mediated by dynamic cellular protusions such as filopodia, which, importantly, could extend the distance over which signalling occurs ${ }^{91-94}$. The nature of the cell-cell contacts could therefore have important consequences for the functional outcomes of signalling (Figure 3a).

Adherens junctions (AJs) mediate cell-cell adhesion and are important for effective Notch signalling in some contexts. Thus, during vertebrate neurogenesis, the dimensions of the contacts made by the 'end-feet' and the integrity of their AJs are important for nascent neurons to engage in effective DLL-Notch signalling with their progenitors to prevent them differentiating. Disrupting the AJs down regulated Notch signalling and caused precocious neurogenesis ${ }^{95}$, whereas manipulations that expanded the size of the apical domain enhanced signalling and reduced neurogenesis ${ }^{96}$.

Strong adhesion between posterior lateral mesoderm and somite cells, mediated by the junctional adhesion molecules Jam1a and Jam2a, is also important for generating a sufficiently high Notch signal to specify the haematopoietic lineage in zebrafish ${ }^{97}$. The contact surface area between migrating lateral mesoderm cells and the somite was decreased when jam1a or jam2a was depleted, and correlated with decreased activation of Notch signalling. As this phenotype could be rescued by widespread expression of ligands, the transduction pathway remained functional, leading to the model that dimensions or stability of contacts was important. The deployment of specific adhesion molecules can thus create a unique topological opportunity for signalling to occur.

Signalling might not always require stable cell-cell contacts, transient interactions can be sufficient to deliver Notch activity and switch cells to a specific cell fate. Neural crest cells expressing DLL could elicit signalling in myotome Notch1containing cells that they contacted, in a ' kiss and run' mode ${ }^{98}$. Likewise, during angiogenic sprouting, because cells constantly shuffle their positions, a presumptive stalk cell might encounter high levels of Notch for only a brief period before losing contact ${ }^{99}$ (Figure $3 b$ ). Transient structures may also extend the range over which a cell can signal. For example, filopodia extend from Dl-producing cells on the $D$. melanogaster notum and their disruption (by specific genetic mutations) perturbed the spacing of sensory organ precursor cells (Figure $3 \mathbf{b}$ ) ${ }^{91}$. Similarly, dynamic filopodia project between neurogenic progenitors and radial glial cells during signalling in the mammalian neocortical progenitor cell niche, although their functional relevance has not yet been tested ${ }^{100}$. And during the formation and maintenance of pigmented stripes in zebrafish, signalling is mediated via long cellular protrusions that extend between xanophores and melanophores. Interestingly the geometry switches: first the protusions carry vesicles of DLL from xanophores to promote melanophore stripe consolidation ${ }^{93}$ (Figure 3 b). Later, in adults, the melanophores extend protusions towards interstripe DLL expressing xanthophores to receive signals necessary for their own survival ${ }^{94}$.

Filopodia-like protusions are more transient than AJs, indicating that a prolonged stable contact point might not be essential for ligand-receptor signalling, although the extent of their contribution to Notch signalling remains to be determined. Modelling experiments suggest that the strength of the signal mediated through 
filopodia will depend principally on the diffusion of ligands or receptors ${ }^{90}$, so that factors that regulate their trafficking would be influential. Furthermore, it is possibile that the types of downstream response might differ according to the way the ligand is presented, - for example, filopodia might provide a burst of signal whereas more stable cellular junctions could generate a more sustained signal.

\section{Nuclear context [L1 heading]}

The output of Notch signalling primarily relies on NICD entering the nucleus; mechanisms that set the nuclear context are therefore critically important as they determine the gene expression programme and consequent physiological outcome. For example, in the outer proliferation centre of the $D$. melanogaster optic lobes, Notch signalling induces neurons to die or to survive, depending on the transcription factors present ${ }^{101}$. Likewise, the contrasting oncogenic and tumor suppressor roles of Notch activity that occur in different tissue contexts are likely the consequences of differential cell-type specific transcriptional programmes ${ }^{102}$. Nuclear mechanisms that confer such context-specific Notch responses are potentially of broad relevance to other signalling pathways, although the precise components might differ.

\section{Key features underpinning the nuclear response [L2 heading]}

A key aspect of specificity will logically result from the presence of cell-type-specific or stage-type-specific transcription factors that alter the selection of responsive genes. Indeed, if CSL itself could function as a 'pioneer', binding to sites in dense nucleosome-covered 'closed' enhancers, it would be difficult to account for the celltype specificity of responsive genes - how, then, could cell death genes be selected only in specific neuronal progeny to correctly programme neural networks, for example? ${ }^{101}$. In agreement with this notion, CSL has a higher affinity for motifs at the edge of nucleosomes, suggesting it binds preferentially to open chromatin ${ }^{103}$. Furthermore, the motifs bound by CSL in Drosophila cells are located preferentially within regions of primed or active chromatin, making it likely that response specificity is aided by many motifs being hidden in chromatin environments that are not accessible to $\mathrm{CSL}^{104}$ (Figure 4). However, even though the NICD-CSL complex prefers ready-primed chromatin regions, it is not just an idle passenger - the consequences of its recruitment include large-scale changes in histone acetylation, removal of repressive complexes and enhanced accessibility of the DNA ${ }^{104-108}$ (Figure 4).

The original 'switch' model proposed that, prior to Notch activation, CSL was present at enhancers in a complex with co-repressors that kept the enhancer silenced by recruiting histone deacetylases or other modifying enzymes ${ }^{109}$. NICD was thought to displace the co-repressors from CSL to render the target enhancers active.

However, this model in its simplest form has been challenged. First, the corepressors KyoT2 and MINT bind to CSL with similar affinities as NICD does, making it hard to explain how NICD could displace them ${ }^{110,111}$. Resolving this question will however also require detailed knowledge of the relative stoichiometries of all the proteins concerned, including NICD. Second, CSL binding dramatically increases at target enhancers following Notch activation, arguing that this event is quite dynamic 105, 112-114. Thus, it is likely that an exchange of entire complexes (rather than coactivators substituting for co-repressors on DNA-resident CSL) occurs, similar to the model for hormone receptors, with the NICD-CSL complex being 'captured' to increase the amount or duration of its binding (Figure 4).

Although further studies are needed to determine the extent to which enhancers are 'marked' by CSL prior to Notch activation, CSL-binding co-repressors are nevertheless likely to contribute to the regulatory landscapes. First, loss or down- 
modulation of CSL leads to derepression of tumour-promoting genes in several contexts ${ }^{115,116}$. Second, as several co-repressors bind directly to CSL in a manner that would preclude concomitant NICD binding, they will titrate the availability of CSL and hence set a threshold that NICD would need to exceed in order to activate transcription ${ }^{110,111,117-119}$. Finally, the different types of co-repressor might give rise to different types of repression complex, especially if they differ in their ability to recruit chromatin-modifying complexes (Box 2). Thus, by binding to their target sites transiently, certain types of CSL-repressor complex might help to make the enhancers more refractory to the effects of NICD. Uncovering their contributions will be important for deciphering the regulatory landscapes that NICD encounters.

\section{Cooperation with transcription factors}

To ensure that the appropriate tissue-/cell-type response occurs, mechanisms that direct NICD to the appropriate enhancers must exist. Although the execution of some roles of Notch signalling relies on a set of common targets, the HES family of bHLHcontaining proteins, other roles depend on diverse transcriptional responses ${ }^{120}$. These responses are likely to be achieved through close cooperation between CSL complexes and other transcription factors (Figure 5), either because specific configurations of binding motifs allow direct interactions between transcription factors and NICD-CSL or because nearby motifs recruit transcription factors that help recruit NICD-CSL indirectly by modifying chromatin. At the same time, other transcription factors might block CSL recruitment to specific enhancers.

One well-characterized example of an enhancer 'signature' is the so-called SPS+A site in Notch-regulated genes during neural precursor specification. This signature combines a pair of specifically orientated CSL motifs (known as SPS) with a binding site for the pro-neural basic helix-loop-helix protein Daughterless (referred to as A) and allows NICD-CSL to interact directly with Daughterless, thereby conferring Notch-bHLH synergy ${ }^{121}$. Similarly, in Caenorhabditis elegans, the ref-1 enhancer contains four predicted binding sites for GATA transcription factors that are required for Notch-dependent endodermal expression ${ }^{122}$ and that probably facilitate NICDCSL recruitment via a direct interaction between CSL and the GATA factor. A third example involves the mouse Foxp3 gene, in which an overlapping CSL-nuclear factor $\mathrm{KB}(\mathrm{NF}-\mathrm{KB})$ binding site within the promoter facilitates cooperative regulation by Notch3 and canonical NF-kB signalling ${ }^{123}$.

By contrast, other examples indicate that cooperation can occur without a distinctive enhancer signature. An association between Runx proteins and NICD-CSL complexes exists in several cellular contexts, with CSL-bound regions enriched for Runx sites ${ }^{105,108,124}$ but without any very precise arrangement of their motifs. Runx proteins are required for CSL to be recruited to these enhancers and it seems likely that the mechanism involves changes in the local chromatin organization to 'expose' the CSL-binding motifs ${ }^{104}$ (Figure 4). Other examples in which specific transcription factors are important exist (see Box 2), but it is as yet unclear whether direct or indirect mechanisms are involved and it remains to be determined whether subsets of transcription factors have a special relationship with NICD-CSL because they introduce a specific partner or chromatin conformation or, alternatively, whether any transcription factor binding in proximity to a CSL-binding motif might be sufficient to render an enhancer responsive.

Notably, binding of specific transcription factors can also prevent enhancers from responding to Notch. A well-characterized example is the zinc-finger transcription factor Ikaros, which restricts the Notch responsiveness of many T-cell targets, including Hes1 and Myc, by binding to their enhancers ${ }^{125-127}$ (Figure 4). In the 
absence of Ikaros, Notch target genes that are normally shut off in thymocytes were persistently expressed and other normally inactive or weakly upregulated genes became strongly induced by Notch ${ }^{125}$. Conversely, re-expressing Ikaros could repress Notch1 target genes, including Myc, in T-cell acute lymphoblastic leukaemia (T-ALL) cells ${ }^{127}$. Among other transcription factors that inhibit the activity of NICDCSL complexes, several do so by binding to the complex rather than by blocking enhancer binding ${ }^{128,129}$. For example, the transcriptional repressor BCL6 inhibits the expression of ESR1 ${ }^{130}$ either by preventing the recruitment of Mam to NICD or by recruiting the histone deacetylase SIRT1 to promote deacetylation of neighbouring histones ${ }^{131}$. Finally, BEN-SOLO proteins can bind to nearby sites on DNA and directly contact CSL to antagonize Notch activity during neurogenesis ${ }^{132}$. Direct and indirect negative regulators are therefore likely to have widespread roles in setting the transcriptional landscape.

\section{Chromatin context and indirect mechanisms [L2 heading]}

If target enhancers need to be present in the appropriate chromatin complex to be bound by CSL, epigenetic mechanisms that alter the accessibility of enhancers should have an important influence on gene expression in responding cells ${ }^{133}$, especially during developmental (and other) transitions when the Notch-responsive programme needs to change between one state and the next (Figure 5). Several examples illustrate that such transitions might be coordinated by stage-specific transcription factors in conjunction with chromatin-modifying complexes. For example, Hamlet/Evi1, when recruited to targets in nascent $D$. melanogaster olfactory neurons, appears to enable a modified response in a subsequent round of Notch signalling by altering histone methylation and density to erase the Notch state inherited from the parental cell ${ }^{134}$. BCL6 similarly mediates stable epigenetic repression of Hes 5 by recruiting SIRT1 during the neurogenic transition in mouse cortical progenitors ${ }^{131}$. Finally, Pax6/Eyeless blocks the ability of NICD to promote tumours in older generation progenitors in some $D$. melanogaster neural stem cell lineages, where it prevents transcriptional activation of direct target genes possibly through BRG1-associated factor (BAF)-SWI/SNF-related chromatin remodelling complex ${ }^{135}$.

Polycomb complex-mediated silencing of target enhancers is also likely to shape the Notch response. Although their action is reversible, two multiprotein Polycomb repressive complexes (PRCs) confer heritable repressive states, and their presence at many Notch-regulated genes in embryonic stem cells indicates their potential importance ${ }^{133}$. The inability of Notch to drive cardiac regeneration in adult rat myocardiocytes is also attributed to PRC-mediated repression ${ }^{136}$. Likewise, the activity of PRCs curtailed NICD-mediated activation of target genes in $D$. melanogaster cells and in human T-ALL cells ${ }^{107,137 .}$

Many other chromatin regulatory complexes have been found to influence Notch activity in vivo (see Box 2). Although the functional data indicate that these complexes contribute to the landscape of target genes, it remains challenging to distinguish whether they do so via specific or non-specific mechanisms.

Nevertheless, as Notch signalling is sensitive to changes in the activity of several chromatin-regulatory complexes, chromatin organization might be particularly significant for NICD responsiveness.

\section{Factors that modify the stability or activity of NICD [L2 heading]}

Another potentially potent way to regulate the Notch pathway is by modulating the activity or stability of NICD (Figure 4). Relatively little is known about these aspects of its regulation post cleavage, but NICD could be subject to post-translational modification or might interact with other proteins that modulate its nuclear levels 
and/or activity. Yes-associated protein (YAP, a key effector of the Hippo tumour suppressor pathway) and Smad3 (an intracellular transducer of transforming growth factor-ßsignals) both augmented the activity of NICD independently of DNA binding 138139

In some contexts, the stability of NICD is affected by its interactions with F-box and WD repeat domain-containing 7 (FBW7), the substrate-recognition component in a ubiquitin ligase complex. Cancer-associated mutations in FBW7 were identified in patients with $\gamma$-secretase insensitive T-ALL and correlated with increased levels of NICD activity ${ }^{140}$. FBW7 has been shown to bind directly to NICD, promoting its polyubiquitylation and proteasomal degradation ${ }^{140,141}$, and, as this interaction is regulated by CDK8 (a nuclear serine/threonine kinase that functions as a transcriptional regulator), it was thought to terminate NICD activity at its transcriptional targets ${ }^{142}$. However, FBW7 affects many other substrates ${ }^{143}$ and regulates the association of CDK8 with the Mediator complex ${ }^{144}$, making it tricky to distinguish the significance of direct NICD regulation in many contexts. Furthermore, although FBW7 mutation affects several developmental processes linked to altered Notch 1 activity in vertebrates ${ }^{145,146}$, mutations in the $D$. melanogaster homologue have not uncovered an equivalent role. Thus, the extent of direct NICD regulation by FBW7 remains to be clarified, and the existence of other E3 ligases that perform similar roles to terminate NICD activity merits further exploration.

SIRT1 and coactivator-associated arginine methyltransferase 1 (CARM1) are speculated to act as rheostats by modulating the activity of NICD. SIRT1 directly associates with NICD and attenuates Notch activity in zebrafish endothelial cells ${ }^{147}$, and CARM1 methylates NICD and regulates the duration of transcriptional responses from some target enhancers ${ }^{148}$. Other modifications may attenuate the transcriptional activity of NICD or affect its nuclear localization. These include hydroxylation, mediated by factor inhibiting hypoxia-inducible factor $(\mathrm{FIH})$ in response to changes in oxygen levels during myogenesis ${ }^{149}$; phosphorylation, by glycogen synthase kinase-3 (GSK-3) ${ }^{150}$ or AKT ${ }^{151}$; and ubiquitylation, conferred by the HECT ubiquitin ligase WWP2 ${ }^{152}{ }^{153}$. Post-translational modifications targeted to positions within the six ankyrin repeats can prevent NICD from forming its tripartite activation complex with CSL and Mam ${ }^{154-156}$. If the relevant enzymes were recruited to specific targets, these modifications could nevertheless lead to differential effects on gene expression. Modifying enzymes potentially provide mechanisms of cross-talk with other pathways, as suggested by the purification of multiple kinase with NICD ${ }^{157}$, and are, in some cases, specific to one paralogue. Clearly, more knowledge about when and where NICD is modified and what effects each modification has on its activity will be important for understanding its operations in a given milieu.

\section{Network context [L1 heading]}

Interpreting the context-specific effects of Notch will ultimately require that we understand the wiring of the regulatory networks in which it operates. Although this presents an enormous challenge, indications already exist as to how differences between cell types affect Notch pathway outcomes, as illustrated by the examples below.

Feedback regulation of ligand expression is one example in which the regulatory logic has profound consequences. For contexts in which classical lateral inhibition occurs, Notch activation frequently inhibits ligand expression to polarize the signalling (negative feedback). This mechanism relies on the HES family of direct Notch targets, which antagonize the activity of proneural bHLH transcription factors, which themselves promote the expression of ligands and E3 ligases (such as Neur) 158. Thus, Notch activation leads to decreased ligand expression, and the fates of the 
cells becomes mutually exclusive ${ }^{5}$. For contexts in which signalling is inductive, Notch activity promotes ligand expression (positive feedback). This feed-forward positive regulation of ligand expression occurs at the signalling boundary in the $D$. melanogaster wing disc and during the formation of sensory patches in the chick ear $24,31,45,159$. In $D$. melanogaster the evidence points to a direct regulation of Ser expression by NICD-CSL ${ }^{9,160}$. By intensifying and perpetuating ligand expression, such positive feedback can sharpen the boundaries between expressing and nonexpressing regions. Alternatively, under some circumstances it could lead to a shutdown if the increased ligand levels blocked signal reception through cis-inbibition. Differences in the wiring of the regulatory network also account for opposing effects on PTEN (phosphatase and tensin homologue) that occur in response to Notch activity. In T-ALL cells, Notch activity, by directly targeting HES1, inhibits the expression of PTEN, thereby promoting proliferation ${ }^{161}$. By contrast, in stalk cells, PTEN is itself a direct target of Notch and is up-regulated to inhibit proliferation ${ }^{162}$.

Differences in the mode of crosstalk between the Notch and epidermal growth factor receptor (EGFR)-Ras pathways lead to them functioning antagonistically in some contexts and cooperatively in others ${ }^{163}$. Such polarized differences partly arise from the regulatory logic of the target enhancers and partly as a consequence of whether Notch regulates the expression of Ras pathway inhibitors or activators (and vice versa). For example, in the D. melanogaster eye, EGFR and Notch pathways cooperate to promote the development of cone cells by converging on enhancers of key differentiation genes (for example, Pax ${ }^{164}$ ), but antagonize one another at the onset of ommatidial development because EGFR promotes the expression of the proneural protein Atonal whereas Notch activity inhibits atonal expression through HES targets ${ }^{165}$. Similarly, during C. elegans vulval development, EGFR activity first initiates Notch activity by upregulating ligand expression to stimulate the receptor on adjacent cells, then Notch activity antagonizes EGFR-Ras signalling by promoting the expression of pathway inhibitors ${ }^{166}$. These examples illustrate how differences in wiring can profoundly influence the consequences of activating the two pathways. Similar differences in crosstalk are likely to underpin many of the context-specific interactions of Notch with Wnt, fibroblast growth factor, Hippo and other pathways.

\section{Conclusions and perspectives}

The ability of the Notch pathway to carry out many tasks despite the relative simplicity of its core pathway relies on the deployment of different levels of control that adapt the pathway to each context. For example, the expression patterns of Notch and its ligands and the tissue architectures can determine both the range and strength of Notch signalling, whereas the nuclear context will shape the identity of the target genes regulated and hence the transcriptional outcome. Many of the strategies, especially in the nuclear context, will be relevant for other signalling pathways that similarly induce a diversity of tasks. For example, the contextdependent modifiers of $\mathrm{Wnt} / \mathrm{\beta}$-catenin signalling that contribute to its differing effects in stem cells include the cocktail of cooperating transcription factors that are present 167. However, other strategies are more likely to be unique to Notch. Notably, the fact that Notch ligands are transmembrane proteins constrains the range of the signal and makes the cell architecture and tissue organization particularly important features. The one-to-one interaction between ligand and receptor places more emphasis on the precise relationship between their levels, especially as they can also inhibit one another when present in the same cells. All the levels of regulation can be modulated to enable the pathway to adapt to a changing environment. However, although we can appreciate how the regulation might occur conceptually, many aspects are still poorly understood, making it hard to predict how physiological and environmental differences will influence signalling. 
Recent progress has been driven by structural studies of key complexes involved in Notch signalling; the next challenge will be to find ways to view the molecules in action, to find where on/in the cell they interact and to discover the levels, stoichiometries and dynamics of the different complexes. A more quantitative picture will aid predictions about the transcriptional and physiological outcomes. Discovering how these can be modulated by environmental factors will also be important for understanding disease susceptibilities from heterozygous mutations in Notch pathway genes. 


\section{Box 1: Mechanistic features of Notch signalling}

All canonical Notch ligands are transmembrane proteins (apart from some unusual relatives in C. elegans) that share a largely similar structure, with an extracellular domain comprised primarily of multiple EGF repeats (EGFR, see figure, which shows D. melanogaster Notch and its ligands, Serrate and Delta). Serrate and its' Jagged orthologues also contain a cysteine rich domain, CRD. Binding by canonical Notch ligands involves the extracellular Delta/Serrate/Lag-2 (DSL) domain and aminoterminal (NT) domain (see figure), which contact EGF repeats 11-12 within the extracellular domain of Notch ${ }^{22,}{ }^{68}$. As the NT domains have phospholipid-binding characteristics ${ }^{169}$, interactions with the adjacent cell membranes might also be involved. Notch receptors on the cell surface are heterodimers (see figure): the two heterodimeric portions (HDN, HDC) interact and together with the cysteine-rich Lin12/Notch repeats (LNRs) form the negative regulatory region (NRR), which occludes the cleavage site for ADAM proteases ${ }^{56}$. The key step induced by ligandbinding is the exposure of this cleavage site, which allows access by proteases. ADAM10 is likely to be the main protease responsible for cleavage under physiological conditions ${ }^{170}$. Cleavage renders the remaining transmembraneintracellular fragment a substrate for the $\gamma$-secretase complex, which catalyses intramembrane proteolysis to release the Notch intracellular domain (NICD). NICD is characterized by a RAM (RBP-J-associated module) domain and ankyrin (ANK) repeats (see figure), both of which are required for interactions with the DNA-binding protein CSL (CBF1/Suppressor of Hairless/Lag-1; also known as RBPJ) ${ }^{3,171-173}$. Near the carboxyl terminus is a PEST domain (see figure), which regulates NICD degradation. Between the ANK repeats and PEST, NICD also contains several nuclear localization signals and a region that can confer transactivation. The association of NICD with CSL forms an interface to which the amino terminus of the co-activator Mastermind (Mam) binds, locking the complex into its active conformation and promoting gene transcription ${ }^{172,173 .}$

\section{Box 2: Players in the nuclear arena Nuclear activation complexes}

The CSL/NICD/MAM complex recruits p300/CBP, which modifies chromatin at target enhancers. Notch dependant histone modifications include wide-spread increases in $\mathrm{H} 3 \mathrm{~K} 27 \mathrm{ac}$ and H3K56ac and a decrease in H3K27me3 ${ }^{104,105}$ 107. Other components that have been associated with the co-activator complex include the demethylase JMJD3 ${ }^{107}$, the RNA helicase Ddx5, the long non-coding RNA (IncRNA) steroid receptor coactivator (SRA) ${ }^{174,175}$. Chromatin modifiers that enhance Notch transcriptional activity include BRG1/Brahma complexes, BRD4, Bre1/RNF40 ${ }^{176}$

Nuclear co-repressor complexes: CSL-binding co-repressors for which direct interactions have been mapped include MINT/SHARP (mammals ${ }^{110}$ ) KyoT2 (mammals; ${ }^{111}$ ), Hairless (Drosophila; ${ }^{118}$ ). SMRT/SMRTR, SIR interactions have also been detected ${ }^{119}$. Co-repressors recruit enzyme complexes that modify chromatin at target enhancers, including class 1 histone deacetylases (HDACs) and histone demethylases Kdm5/Lid and LSD1 117, 119, 157, 177, 178. Histone chaperone complexes containing CAF1, NAP1, Asf1 are also implicated in CSL dependant repression of target enhancers ${ }^{119}$. In some cases, co-repressor-recruitment of modifying enzymes relies on intermediaries, including Groucho/TLE, CtBP ${ }^{179}$.

\section{Additional examples of Co-operating TFs}

TEAD4: a DNA binding protein that is regulated by the Hippo pathway, TEAD4 cobinds the enhancer of Cdx2 with Notch/CSL in early mouse embryos ${ }^{180}$. 
SoxF: Combinatorial regulation by SoxF TFs is necessary for Dll4 expression during arterial specification and co-binding is needed to give full enhancer activity ${ }^{181}$.

TCF: TCF sites are present in enhancers collaboratively regulated by Notch1 and Wnt activity in intestinal crypts ${ }^{182}$.

ETS: Ets1 binding motif and ETS1 occupancy was enriched near NICD/CSL bound regions in T-ALL cells ${ }^{176}$ 


\section{Figure Legends:}

Figure 1 | Ligand binding leads to exposure of the cleavage site in Notch (a) Summary of core pathway: when canonical Notch ligands (green) bind to Notch receptors (purple; orange indicates EGF repeats 11-12, pink indicates the Negative Regulatory Region, NRR) on the adjacent cell surface they elicit two proteolytic cleavage events, the first by ADAM10 and the second by y-secretase, that release the Notch intracellular domain (NICD). In the nucleus NICD interacts with the DNAbinding protein CSL (CBF1/Suppressor of Hairless/Lag-1; also known as RBPJ) ${ }^{3,171-}$ 173 and the co-activator Mastermind (Mam) to promote gene transcription ${ }^{172,173}$. (b) Schematic based on the crystal structure ${ }^{56,57}$ illustrating how the Notch-NRR occludes the cleavage site for ADAM proteases until its is exposed by forces generated through ligand-binding. Left panel: the NRR comprises 3 Lin12/Notch repeats (LNR, pink) and the heterodimerization domain (HD; dark blue and light blue), which surround the recognition site making it in accessible to ADAM proteases. Also represented are 3 EGF-repeats from the Notch ECD, the RAM (RBP-J-associated module) and first ankyrin repeat (ANK1) domain in NICD. Right panel: Ligand binding exerts a force on the receptor (right panel), which displaces the LNRs, exposing the site for cleavage by ADAM 10. This cleavage renders the residual transmembrane Notch fragment a substrate for proteolyisis by the $\gamma$ secretase complex, to release NICD (see a)

\section{Figure 2 | The consequences of cis inhibition and Fringe expression on Notch signalling}

(a) Relative levels of ligands (green) and Notch receptors (purple) determine whether cells send or receive signals because cis interactions between ligands and receptors present on the same cells are inhibitory (light shading). Receiver cell, left, expresses more Notch than Delta $(\mathrm{N}>\mathrm{DL})$; some Notch molecules are cis-inhibited by DL but sufficient Notch remains available to interact with ligands from neighbouring cells, making the cell capable of receiving signals. Sending cell, right, expresses more DL than Notch (DL>N); all Notch molecules are cis-inhibited by DL and sufficient DL remains available to interact with receptors on neighbouring cells, making the cell capable of sending a signal.

(b) Fringe proteins (yellow) glycosylate the Notch extracellular domain and modulate both cis and trans interactions with ligands. In the absence of Fng (left) Jag preferentially cis-inhibits Notch, so that none of the ligand is available for signalling. Any uninhibited Notch is competent to interact with either DL or Jag ligands from neighbouring cells. When Fng is present (right) glycosylation of Notch interferes with Jag (turquoise) cis and trans interactions, with the result that the cell can only receive signals from DL ligands (green), but can now send Jag signals.

Figure 3 | Influence of cell contacts and tissue architecture on signalling (a) Contact-dimensions between Delta- (green) and Notch-expressing cells (purple) could alter Notch responses. Left: Large contact region, many ligand-receptor interactions occur generating high/prolonged NICD (dark purple/Response 1). Middle: Smaller contact surface, fewer ligand-receptor interactions, less NICD and different/fewer target genes activated (mid-purple/Response 2). Right: Cell contacts via filopodia have limited/transient receptor-ligand interactions, generating low/transient NICD and response (pale purple/Response 3).

(b) Notch signalling associated with different cell architectures. Angiogenic branching and extension of blood vessels involves the formation of dynamic contacts between tip cells (green, DIl4-producing) and adjacent stalk cells (mauve, Notch expressing), during which a presumptive stalk cell might receive high levels of Notch for a brief period before losing contact. When Notch signalling is perturbed, excess tip cells 
generate a densely branched, compacted network. Selection of sensory organ precursor (SOP) cells involves direct contact between signal-sending cells (green) and signal-receiving cells (purple), but might also require filipodia to transduce the signal across a longer range. Perturbations to Notch give tufts of bristles, as all neighbouring cells become SOPs. Disruptions to filopodia result in extra SOPs and altered bristle spacing. During the formation of pigmented stripes in zebrafish, melanophores are restricted to stripes via signals conveyed by long cellular protrusions extending from xanthophores (green). Perturbations to Notch result in expansion of melanophores into the inter-stripe region.

\section{Figure 4 | Regulation of the nuclear context}

Steps involved in NICD-mediated activation at target enhancers, indicating the types of regulation occurring at each; left side, Inhibitory mechanisms (grey); right side, positive mechanisms (turquoise, purple). In the absence of cooperating transcription factors, CSL motifs (orange) are obscured by nucleosomes; this may be brought about by the presence of specific repressors (e.g. Hamlet/Evi, grey) acting in combination with chromatin modifying/remodelling enzymes. Co-operating transcription factors: (e.g. Runx, cyan) promote chromatin remodelling to expose CSL motifs. In many contexts CSL binding remains transient/unstable under these conditions, possibly due to the influence of its co-repressors (dark blue). Presence of NICD: a tertiary complex containing CSL, NICD and Mam is formed and resides at the CSL binding-sites, where it recruits co-activators and stimulates transcription. Levels/extent/duration of transcription will depend on the levels/perdurance of NICD, which is regulated by post-translational modifications (PTMs). Ubiqutination directed by E3 ligases, via intermediaries such as Fbw7, promote degradation to terminate NICD activity. Note that some target genes may already be transcribed in the absence of Notch, so that Notch binding will augment rather than initiate expression.

\section{Figure 5 | Transitions in Notch-responsive programmes}

Requirement for different co-operating factors and repressors to bring about changes in the Notch response at different developmental/physiological transitions. Top: In a nucleus that contains no co-operating TFS, CSL motifs (brown) are inaccessible. Middle: when an enabling co-operating TF is expressed (Cooperating TF1, cyan), it binds to target sites in enhancers making them competent to respond to CSL-NICD complexes, yielding a specific Notch response 1 . When the cell undergoes a subsequent transition (e.g. following cell division in the olfactory lineage) different TFs are expressed, some of which inhibit (grey hexagon) the response 1 class of genes by conferring a non-permissive chromatin context and others (co-operating TF2, green) opening up a new cohort of enhancers to enable a different Notch response (Notch response 2). In this way, nuclei can transition from one response to another depending on time, other signals.

\section{Acknowledgements}

The author apologizes for the many articles that could not be cited owing to space limitations. She also thanks Maria Gomez-Lamarca for help in preparing the Figures and Agnes Asselin for the drawings in Figure 3. Work in the Bray lab is supported by a programme grant from the Medical Research Council as well as by funding from Biology and Biotechnology Research Council. 


\section{Glossary terms:}

EGF-LIKE REPEATS: Protein domains, commonly found in the extracellular domain of membrane-bound proteins, that are related to a sequence in EGF and include cysteine residues involved in disulphide bonds. EGF-like domains frequently occur in numerous tandem copies in proteins as in Notch (EGF-like_dom: IPR000742)

PARALOGUES Sequences, or genes, that have originated from a common ancestral sequence, or gene, by a duplication event.

LATERAL INHIBITION The process by which a cell with a particular fate interacts with its immediate neighbours to prevent them from adopting the same fate.

GROWTH ORGANIZER Group of cells that produces signals necessary to promote growth of a tissue.

SOMITOGENESIS The process by which somites, blocks of mesoderm that give rise to axial muscles, bones and dermis in vertebrates, are formed.

PLACODE Ectodermal thickening from which a sense organ or ganglion develops.

ESCRT (endosomal sorting complex required for transport). The multiprotein ESCRT machinery (ESCRT-I, -II and -III) promotes inward vesiculation at the limiting membrane of the sorting endosome, and selects cargo proteins for delivery to the intralumenal vesicles of multivesicular bodies.

SOP CELL Sensory organ precursor cell that gives rise to all cells in a Drosophila sensory organ.

ADHERENS JUNCTIONS Actin-filament-associated, epithelial cell-cell junctions that have classical cadherins as their core component.

END FEET The name given to the apical membrane surface as a consequence of cortical neuroepithelial progenitors becoming very tall and thin over the course of development.

ANGIOGENIC FRONT Region at the leading edge of a vascular network where tip cells are located to initiate further growth and branching of the network

FILOPODIA Thin cellular processes containing long, unbranched, parallel bundles of actin filaments.

NOTUM structures that are part of the back of an animal, in insects the back of the thorax.

XANTHOPHORES Yellow chromatophores, pigment-containing and light-reflecting cells, of a fish, amphibian, or reptile.

MELANOPHORES Melanin containing cells, of a fish, amphibian, or reptile that appear black or dark-brown because of melanin's light absorbing qualities.

ENHANCER A DNA segment that increases transcription of a linked promoter if placed in either orientation, upstream or downstream. 
BASIC HELIX-LOOP-HELIX (bHLH) GENES Genes that encode proteins that contain a basic domain adjacent to two $\alpha$-helices separated by a loop (the HLH domain), which binds DNA in a sequence-specific manner.

HES gene family A family of genes related to $\underline{H}$ airy and Enhancer of $\underline{s} p l i t$ that encode nuclear proteins that suppress transcription.

GATA-TYPE TRANSCRIPTION FACTORS A family of transcription factors that contain a zinc-finger motif that was first identified in the vertebrate GATA1 protein. These transcription factors bind the consensus sequence GATA in the regulatory regions of genes.

ZINC FINGER A motif in proteins that contains conserved cysteine residues. The sulphydryl groups of the cysteines coordinate a $\mathrm{Zn}^{2+}$ ion.

BEN-SOLO PROTEINS Proteins containing only a BEN domain, a sequence-specific DNA-binding domain that has been identified in some transcription repressors.

NEUROGENIC TRANSITION Change in the competence of neural precursor cells that enable them to generate different types of neural or glial progeny

F-BOX PROTEIN A component of the machinery for the ubiquitin-dependent degradation of proteins. F-box proteins recognize specific substrates and, with the help of other subunits of an E3 ubiquitin ligase complex, deliver them to the E2 ubiquitin-conjugating enzyme.

WD40 PROTEIN A 40-amino-acid-long protein motif that contains a WD dipeptide at its carboxy terminus. This domain is found in many functionally diverse proteins and mediates protein-protein interactions.

MEDIATOR COMPLEX A multiprotein complex that is required for gene transcription by RNA polymerase II.

HECT Stands for homologous to E6-AP carboxyl terminus. The HECT domain is a 350-amino-acid domain, highly conserved among a family of E3 enzymes. 


\section{References Cited:}

Highlighted are recent references that illustrate points of interest for those entering the field

1. Artavanis-Tsakonas, S., Rand, M.D. \& Lake, R.J. Notch signaling: cell fate control and signal integration in development. Science 284, 770-6 (1999).

2. Bray, S.J. Notch signalling: a simple pathway becomes complex. Nat Rev Mol Cell Biol 7, 678-89 (2006).

3. Kopan, R. \& Ilagan, M.X. The canonical Notch signaling pathway: unfolding the activation mechanism. Cell 137, 216-33 (2009).

4. Kitagawa, M. Notch signalling in the nucleus: roles of Mastermind-like (MAML) transcriptional coactivators. J Biochem (2015).

5. Collier, J.R., Monk, N.A., Maini, P.K. \& Lewis, J.H. Pattern formation by lateral inhibition with feedback: a mathematical model of delta-notch intercellular signalling. J Theor Biol 183, 429-46 (1996).

6. Vallejo, D.M., Caparros, E. \& Dominguez, M. Targeting Notch signalling by the conserved miR-8/200 microRNA family in development and cancer cells. EMBO J 30, 756-69 (2011).

7. Brabletz, S. et al. The ZEB1/miR-200 feedback loop controls Notch signalling in cancer cells. EMBO J 30, 770-82 (2011).

8. Kim, J., Irvine, K.D. \& Carroll, S.B. Cell recognition, signal induction, and symmetrical gene activation at the dorsal-ventral boundary of the developing Drosophila wing. Cell 82, 795-802 (1995).

9. Yan, S.J., Gu, Y., Li, W.X. \& Fleming, R.J. Multiple signaling pathways and a selector protein sequentially regulate Drosophila wing development.

Development 131, 285-98 (2004).

10. Shimojo, H. et al. Oscillatory control of Delta-like1 in cell interactions regulates dynamic gene expression and tissue morphogenesis. Genes Dev 30, 102-16 (2016). Recent paper demonstrating Delta oscillations in neural stem cells.

11. Bone, R.A. et al. Spatiotemporal oscillations of Notch1, DII1 and NICD are coordinated across the mouse PSM. Development 141, 4806-16 (2014).

12. Wahi, K., Bochter, M.S. \& Cole, S.E. The many roles of Notch signaling during vertebrate somitogenesis. Semin Cell Dev Biol (2014).

13. Kiernan, A.E., Xu, J. \& Gridley, T. The Notch ligand JAG1 is required for sensory progenitor development in the mammalian inner ear. PLoS Genet 2, e4 (2006).

14. Neves, J., Parada, C., Chamizo, M. \& Giraldez, F. Jagged 1 regulates the restriction of Sox2 expression in the developing chicken inner ear: a mechanism for sensory organ specification. Development 138, 735-44 (2011).

15. Haddon, C., Jiang, Y.J., Smithers, L. \& Lewis, J. Delta-Notch signalling and the patterning of sensory cell differentiation in the zebrafish ear: evidence from the mind bomb mutant. Development 125, 4637-44 (1998).

16. Brooker, R., Hozumi, K. \& Lewis, J. Notch ligands with contrasting functions: Jagged1 and Delta1 in the mouse inner ear. Development 133, 1277-86 (2006).

17. Neves, J., Abello, G., Petrovic, J. \& Giraldez, F. Patterning and cell fate in the inner ear: a case for Notch in the chicken embryo. Dev Growth Differ 55, 96$112(2013)$.

18. Daudet, N., Ariza-McNaughton, L. \& Lewis, J. Notch signalling is needed to maintain, but not to initiate, the formation of prosensory patches in the chick inner ear. Development 134, 2369-78 (2007). 
19. Benedito, R. \& Hellstrom, M. Notch as a hub for signaling in angiogenesis. Exp Cell Res 319, 1281-8 (2013).

20. Hellstrom, M. et al. DII4 signalling through Notch1 regulates formation of tip cells during angiogenesis. Nature 445, 776-80 (2007).

21. Kopan, R., Chen, S. \& Liu, Z. Alagille, Notch, and robustness: why duplicating systems does not ensure redundancy. Pediatr Nephrol 29, 651-7 (2014).

22. Luca, V.C. et al. Structural biology. Structural basis for Notch1 engagement of Delta-like 4. Science 347, 847-53 (2015). Describes the binding interface between a ligand and Notch that also suggested a contribution from glycosylation

23. Gama-Norton, L. et al. Notch signal strength controls cell fate in the haemogenic endothelium. Nat Commun 6, 8510 (2015). By using different Cre derivatives of Notch1, revealed differences in signalling strengths associated with two outcomes.

24. Petrovic, J. et al. Ligand-dependent Notch signaling strength orchestrates lateral induction and lateral inhibition in the developing inner ear.

Development 141, 2313-24 (2014).

25. Bellavia, D. et al. Notch3: from subtle structural differences to functional diversity. Oncogene 27, 5092-8 (2008).

26. Penton, A.L., Leonard, L.D. \& Spinner, N.B. Notch signaling in human development and disease. Semin Cell Dev Biol 23, 450-7 (2012).

27. Fan, X. et al. Notch1 and notch2 have opposite effects on embryonal brain tumor growth. Cancer Res 64, 7787-93 (2004).

28. Liu, Z. et al. The intracellular domains of Notch1 and Notch2 are functionally equivalent during development and carcinogenesis. Development 142, 2452-63 (2015). Investigates functional differences between Notch1 and Notch 2 by swapping their intracellular domains in the endogenous loci.

29. Liu, Z. et al. The extracellular domain of Notch2 increases its cell-surface abundance and ligand responsiveness during kidney development. Dev Cell 25, 585-98 (2013).

30. del Alamo, D., Rouault, H. \& Schweisguth, F. Mechanism and significance of cis-inhibition in Notch signalling. Curr Biol 21, R40-7 (2011).

31. de Celis, J.F. \& Bray, S. Feed-back mechanisms affecting Notch activation at the dorsoventral boundary in the Drosophila wing. Development 124, 3241-51 (1997).

32. Micchelli, C.A., Rulifson, E.J. \& Blair, S.S. The function and regulation of cut expression on the wing margin of Drosophila: Notch, Wingless and a dominant negative role for Delta and Serrate. Development 124, 1485-95 (1997).

33. Becam, I., Fiuza, U.M., Arias, A.M. \& Milan, M. A role of receptor Notch in ligand cis-inhibition in Drosophila. Curr Biol 20, 554-60 (2010).

34. Glittenberg, M., Pitsouli, C., Garvey, C., Delidakis, C. \& Bray, S. Role of conserved intracellular motifs in Serrate signalling, cis-inhibition and endocytosis. Embo J 25, 4697-706 (2006).

35. Sprinzak, D. et al. Cis-interactions between Notch and Delta generate mutually exclusive signalling states. Nature 465, 86-90 (2010). Elegant modelling experiments suggest how cis-inhibition could make the switch between two mutually exclusive cell states ultra-sensitive.

36. Miller, A.C., Lyons, E.L. \& Herman, T.G. cis-Inhibition of Notch by endogenous Delta biases the outcome of lateral inhibition. Curr Biol 19, 137883 (2009).

37. Boareto, M. et al. Jagged-Delta asymmetry in Notch signaling can give rise to a Sender/Receiver hybrid phenotype. Proc Natl Acad Sci U S A 112, E402-9 (2015). 
38. James, A.C. et al. Notch4 reveals a novel mechanism regulating Notch signal transduction. Biochim Biophys Acta 1843, 1272-84 (2014).

39. Ladi, E. et al. The divergent DSL ligand DII3 does not activate Notch signaling but cell autonomously attenuates signaling induced by other DSL ligands. $J$ Cell Biol 170, 983-92 (2005).

40. Hoyne, G.F., Chapman, G., Sontani, Y., Pursglove, S.E. \& Dunwoodie, S.L. A cell autonomous role for the Notch ligand Delta-like 3 in alphabeta T-cell development. Immunol Cell Biol 89, 696-705 (2011).

41. Chapman, G., Sparrow, D.B., Kremmer, E. \& Dunwoodie, S.L. Notch inhibition by the ligand DELTA-LIKE 3 defines the mechanism of abnormal vertebral segmentation in spondylocostal dysostosis. Hum Mol Genet 20, 905-16 (2011).

42. Takeuchi, H. \& Haltiwanger, R.S. Significance of glycosylation in Notch signaling. Biochem Biophys Res Commun 453, 235-42 (2014).

43. Rana, N.A. \& Haltiwanger, R.S. Fringe benefits: functional and structural impacts of O-glycosylation on the extracellular domain of Notch receptors. Curr Opin Struct Biol 21, 583-9 (2011).

44. Yang, L.T. et al. Fringe glycosyltransferases differentially modulate Notch1 proteolysis induced by Delta1 and Jagged1. Mol Biol Cell 16, 927-42 (2005).

45. Panin, V.M., Papayannopoulos, V., Wilson, R. \& Irvine, K.D. Fringe modulates Notch-ligand interactions. Nature 387, 908-12 (1997).

46. Doherty, D., Feger, G., Younger-Shepherd, S., Jan, L.Y. \& Jan, Y.N. Delta is a ventral to dorsal signal complementary to Serrate, another Notch ligand, in Drosophila wing formation. Genes Dev 10, 421-34 (1996).

47. Benedito, R. et al. The notch ligands DII4 and Jagged1 have opposing effects on angiogenesis. Cell 137, 1124-35 (2009). Example of a context that illustrates divergent consequences from activation by different ligands.

48. D'Amato, G. et al. Sequential Notch activation regulates ventricular chamber development. Nat Cell Biol 18, 7-20 (2016).

49. Marklund, U. et al. Domain-specific control of neurogenesis achieved through patterned regulation of Notch ligand expression. Development 137, 437-45 (2010).

50. LeBon, L., Lee, T.V., Sprinzak, D., Jafar-Nejad, H. \& Elowitz, M.B. Fringe proteins modulate Notch-ligand cis and trans interactions to specify signaling states. Elife 3, e02950 (2014). Modelling experiments that shed light on how Fringes can affect signalling capabilites

51. Parks, A.L., Klueg, K.M., Stout, J.R. \& Muskavitch, M.A. Ligand endocytosis drives receptor dissociation and activation in the Notch pathway. Development 127, 1373-85 (2000).

52. Weinmaster, G. \& Fischer, J.A. Notch ligand ubiquitylation: what is it good for? Dev Cell 21, 134-44 (2011).

53. Fortini, M.E. \& Bilder, D. Endocytic regulation of Notch signaling. Curr Opin Genet Dev 19, 323-8 (2009).

54. Yamamoto, S., Charng, W.L. \& Bellen, H.J. Endocytosis and intracellular trafficking of Notch and its ligands. Curr Top Dev Biol 92, 165-200 (2010).

55. Meloty-Kapella, L., Shergill, B., Kuon, J., Botvinick, E. \& Weinmaster, G. Notch ligand endocytosis generates mechanical pulling force dependent on dynamin, epsins, and actin. Dev Cell 22, 1299-312 (2012).

56. Gordon, W.R. et al. Structural basis for autoinhibition of Notch. Nat Struct Mol Biol 14, 295-300 (2007). Described the structure of the Notch negative regulatory region (NRR), shedding new insight on mechanisms of activation.

57. Gordon, W.R. et al. Mechanical Allostery: Evidence for a Force Requirement in the Proteolytic Activation of Notch. Dev Cell 33, 729-36 
(2015). Elegant experiments showing that application of force could activate the receptor.

58. Wang, W. \& Struhl, G. Distinct roles for Mind bomb, Neuralized and Epsin in mediating DSL endocytosis and signaling in Drosophila. Development 132, 2883-94 (2005).

59. Overstreet, E., Fitch, E. \& Fischer, J.A. Fat facets and Liquid facets promote Delta endocytosis and Delta signaling in the signaling cells. Development 131, 5355-66 (2004).

60. Lai, E.C., Roegiers, F., Qin, X., Jan, Y.N. \& Rubin, G.M. The ubiquitin ligase Drosophila Mind bomb promotes Notch signaling by regulating the localization and activity of Serrate and Delta. Development 132, 2319-32 (2005).

61. Daskalaki, A. et al. Distinct intracellular motifs of Delta mediate its ubiquitylation and activation by Mindbomb1 and Neuralized. J Cell Biol 195, 1017-31 (2011).

62. Le Borgne, R., Remaud, S., Hamel, S. \& Schweisguth, F. Two distinct E3 ubiquitin ligases have complementary functions in the regulation of delta and serrate signaling in Drosophila. PLoS Biol 3, e96 (2005).

63. Fontana, J.R. \& Posakony, J.W. Both inhibition and activation of Notch signaling rely on a conserved Neuralized-binding motif in Bearded proteins and the Notch ligand Delta. Dev Biol 333, 373-85 (2009).

64. Koo, B.K. et al. An obligatory role of mind bomb-1 in notch signaling of mammalian development. PLoS One 2, e1221 (2007).

65. Le Borgne, R. \& Schweisguth, F. Unequal segregation of Neuralized biases Notch activation during asymmetric cell division. Dev Cell 5, 139-48 (2003).

66. Yoon, K.J. et al. Mind bomb 1-expressing intermediate progenitors generate notch signaling to maintain radial glial cells. Neuron 58, 519-31 (2008).

67. Dong, Z., Yang, N., Yeo, S.Y., Chitnis, A. \& Guo, S. Intralineage directional Notch signaling regulates self-renewal and differentiation of asymmetrically

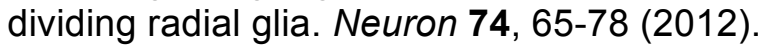

68. Vaccari, T., Lu, H., Kanwar, R., Fortini, M.E. \& Bilder, D. Endosomal entry regulates Notch receptor activation in Drosophila melanogaster. $J$ Cell Biol 180, 755-62 (2008). Takes a systematic approach to assess the contibution made by endocytosis in the signal receiving cell.

69. Giebel, B. \& Wodarz, A. Tumor suppressors: control of signaling by endocytosis. Curr Biol 16, R91-2 (2006).

70. Thompson, B.J. et al. Tumor suppressor properties of the ESCRT-II complex component Vps25 in Drosophila. Dev Cell 9, 711-20 (2005).

71. Troost, T., Jaeckel, S., Ohlenhard, N. \& Klein, T. The tumour suppressor Lethal (2) giant discs is required for the function of the ESCRT-III component Shrub/CHMP4. J Cell Sci 125, 763-76 (2012).

72. Vaccari, T. \& Bilder, D. The Drosophila tumor suppressor vps25 prevents nonautonomous overproliferation by regulating notch trafficking. Dev Cell 9 , 687-98 (2005).

73. Chastagner, P., Israel, A. \& Brou, C. Itch/AIP4 mediates Deltex degradation through the formation of K29-linked polyubiquitin chains. EMBO Rep 7, 114753 (2006).

74. Hori, K., Sen, A., Kirchhausen, T. \& Artavanis-Tsakonas, S. Synergy between the ESCRT-III complex and Deltex defines a ligand-independent Notch signal. J Cell Biol 195, 1005-15 (2011).

75. Shimizu, H. et al. Compensatory flux changes within an endocytic trafficking network maintain thermal robustness of Notch signaling. Cell 157, 1160-74 (2014). 
76. Kechad, A. et al. Numb is required for the production of terminal asymmetric cell divisions in the developing mouse retina. J Neurosci 32, 17197-210 (2012).

77. Frise, E., Knoblich, J.A., Younger-Shepherd, S., Jan, L.Y. \& Jan, Y.N. The Drosophila Numb protein inhibits signaling of the Notch receptor during cellcell interaction in sensory organ lineage. Proc Natl Acad Sci U S A 93, 1192532 (1996).

78. Spana, E.P. \& Doe, C.Q. Numb antagonizes Notch signaling to specify sibling neuron cell fates. Neuron 17, 21-6 (1996).

79. Zilian, O. et al. Multiple roles of mouse Numb in tuning developmental cell fates. Curr Biol 11, 494-501 (2001).

80. Lin, S. et al. Lineage-specific effects of Notch/Numb signaling in postembryonic development of the Drosophila brain. Development 137, 43-51 (2010).

81. Kandachar, V. \& Roegiers, F. Endocytosis and control of Notch signaling. Curr Opin Cell Biol 24, 534-40 (2012).

82. Couturier, L., Mazouni, K. \& Schweisguth, F. Numb localizes at endosomes and controls the endosomal sorting of notch after asymmetric division in Drosophila. Curr Biol 23, 588-93 (2013).

83. O'Connor-Giles, K.M. \& Skeath, J.B. Numb inhibits membrane localization of Sanpodo, a four-pass transmembrane protein, to promote asymmetric divisions in Drosophila. Dev Cell 5, 231-43 (2003).

84. Hutterer, A. \& Knoblich, J.A. Numb and alpha-Adaptin regulate Sanpodo endocytosis to specify cell fate in Drosophila external sensory organs. EMBO Rep 6, 836-42 (2005).

85. Couturier, L., Vodovar, N. \& Schweisguth, F. Endocytosis by Numb breaks Notch symmetry at cytokinesis. Nat Cell Biol 14, 131-9 (2012). Pioneering use of live imaging to track Numb and Notch during cell fate decisions.

86. Coumailleau, F., Furthauer, M., Knoblich, J.A. \& Gonzalez-Gaitan, M. Directional Delta and Notch trafficking in Sara endosomes during asymmetric cell division. Nature 458, 1051-5 (2009).

87. Kressmann, S., Campos, C., Castanon, I., Furthauer, M. \& Gonzalez-Gaitan, M. Directional Notch trafficking in Sara endosomes during asymmetric cell division in the spinal cord. Nat Cell Biol 17, 333-9 (2015).

88. Herranz, H., Stamataki, E., Feiguin, F. \& Milan, M. Self-refinement of Notch activity through the transmembrane protein Crumbs: modulation of gammasecretase activity. EMBO Rep 7, 297-302 (2006).

89. Singh, J. \& Mlodzik, M. Hibris, a Drosophila nephrin homolog, is required for presenilin-mediated Notch and APP-like cleavages. Dev Cell 23, 82-96 (2012).

90. Khait, I. et al. Quantitative Analysis of Delta-like 1 Membrane Dynamics Elucidates the Role of Contact Geometry on Notch Signaling. Cell Rep 14, 225-33 (2016).

91. Cohen, M., Georgiou, M., Stevenson, N.L., Miodownik, M. \& Baum, B. Dynamic filopodia transmit intermittent Delta-Notch signaling to drive pattern refinement during lateral inhibition. Dev Cell 19, 78-89 (2010).

92. Huang, H. \& Kornberg, T.B. Myoblast cytonemes mediate $\mathrm{Wg}$ signaling from the wing imaginal disc and Delta-Notch signaling to the air sac primordium. Elife 4, e06114 (2015).

93. Eom, D.S., Bain, E.J., Patterson, L.B., Grout, M.E. \& Parichy, D.M. Longdistance communication by specialized cellular projections during pigment pattern development and evolution. Elife 4 (2015).

94. Hamada, H. et al. Involvement of Delta/Notch signaling in zebrafish adult pigment stripe patterning. Development 141, 318-24 (2014). 
95. Hatakeyama, J. et al. Cadherin-based adhesions in the apical endfoot are required for active Notch signaling to control neurogenesis in vertebrates. Development 141, 1671-82 (2014).

96. Clark, B.S. et al. Loss of Llgl1 in retinal neuroepithelia reveals links between apical domain size, Notch activity and neurogenesis. Development 139, 1599-610 (2012).

97. Kobayashi, I. et al. Jam1a-Jam2a interactions regulate haematopoietic stem cell fate through Notch signalling. Nature 512, 319-23 (2014).

98. Rios, A.C., Serralbo, O., Salgado, D. \& Marcelle, C. Neural crest regulates myogenesis through the transient activation of $\mathrm{NOTCH}$. Nature 473, 532-5 (2011).

99. Jakobsson, L. et al. Endothelial cells dynamically compete for the tip cell position during angiogenic sprouting. Nat Cell Biol 12, 943-53 (2010).

100. Nelson, B.R., Hodge, R.D., Bedogni, F. \& Hevner, R.F. Dynamic interactions between intermediate neurogenic progenitors and radial glia in embryonic mouse neocortex: potential role in DII1-Notch signaling. J Neurosci 33, 912239 (2013).

101. Bertet, C. et al. Temporal patterning of neuroblasts controls Notch-mediated cell survival through regulation of Hid or Reaper. Cell 158, 1173-86 (2014).

102. Radtke, F. \& Raj, K. The role of Notch in tumorigenesis: oncogene or tumour suppressor? Nat Rev Cancer 3, 756-67 (2003).

103. Lake, R.J., Tsai, P.F., Choi, I., Won, K.J. \& Fan, H.Y. RBPJ, the major transcriptional effector of Notch signaling, remains associated with chromatin throughout mitosis, suggesting a role in mitotic bookmarking. PLoS Genet 10, e1004204 (2014).

104. Skalska, L. et al. Chromatin signatures at Notch-regulated enhancers reveal large-scale changes in H3K56ac upon activation. EMBO J 34, 1889-904 (2015).

105. Wang, H. et al. NOTCH1-RBPJ complexes drive target gene expression through dynamic interactions with superenhancers. Proc Natl Acad Sci U S A 111, 705-10 (2014). Together with 104 demonstrate both that largescale chromatin changes occur at Notch-regulated enhancers and that CSL binding is increased in Notch active cells (along with 112,113)

106. Yashiro-Ohtani, Y. et al. Long-range enhancer activity determines Myc sensitivity to Notch inhibitors in T cell leukemia. Proc Natl Acad Sci U S A 111, E4946-53 (2014).

107. Ntziachristos, P. et al. Genetic inactivation of the polycomb repressive complex 2 in T cell acute lymphoblastic leukemia. Nat Med 18, 298-301 (2012).

108. Hass, M.R. et al. SpDamID: Marking DNA Bound by Protein Complexes Identifies Notch-Dimer Responsive Enhancers. Mol Cell 59, 685-97 (2015).

109. Kao, H.Y. et al. A histone deacetylase corepressor complex regulates the Notch signal transduction pathway. Genes Dev 12, 2269-77 (1998).

110. Collins, K.J., Yuan, Z. \& Kovall, R.A. Structure and function of the CSL-KyoT2 corepressor complex: a negative regulator of Notch signaling. Structure 22, 70-81 (2014).

111. VanderWielen, B.D., Yuan, Z., Friedmann, D.R. \& Kovall, R.A. Transcriptional repression in the Notch pathway: thermodynamic characterization of CSLMINT (Msx2-interacting nuclear target protein) complexes. J Biol Chem 286, 14892-902 (2011).

112. Krejci, A. \& Bray, S. Notch activation stimulates transient and selective binding of $\mathrm{Su}(\mathrm{H}) / \mathrm{CSL}$ to target enhancers. Genes Dev 21, 1322-7 (2007). Together with 113 and 105 demonstrate that CSL binding is dynamic, leading to a questioning of the original "switch models". 
113. Castel, D. et al. Dynamic binding of RBPJ is determined by Notch signaling status. Genes Dev 27, 1059-71 (2013).

114. Housden, B.E. et al. Transcriptional dynamics elicited by a short pulse of notch activation involves feed-forward regulation by $\mathrm{E}(\mathrm{spl}) / \mathrm{Hes}$ genes. PLoS Genet 9, e1003162 (2013).

115. Procopio, M.G. et al. Combined CSL and p53 downregulation promotes cancer-associated fibroblast activation. Nat Cell Biol 17, 1193-204 (2015).

116. Braune, E.B. et al. Loss of CSL Unlocks a Hypoxic Response and Enhanced Tumor Growth Potential in Breast Cancer Cells. Stem Cell Reports 6, 643-51 (2016).

117. Mulligan, P. et al. A SIRT1-LSD1 corepressor complex regulates Notch target gene expression and development. Mol Cell 42, 689-99 (2011).

118. Kurth, P., Preiss, A., Kovall, R.A. \& Maier, D. Molecular analysis of the notch repressor-complex in Drosophila: characterization of potential hairless binding sites on suppressor of hairless. PLoS One 6, e27986 (2011).

119. Borggrefe, T. \& Oswald, F. The Notch signaling pathway: transcriptional regulation at Notch target genes. Cell Mol Life Sci 66, 1631-46 (2009).

120. Bray, S. \& Bernard, F. Notch targets and their regulation. Curr Top Dev Biol 92, 253-75 (2010).

121. Cave, J.W., Loh, F., Surpris, J.W., Xia, L. \& Caudy, M.A. A DNA transcription code for cell-specific gene activation by notch signaling. Curr Biol 15, 94-104 (2005).

122. Neves, A., English, K. \& Priess, J.R. Notch-GATA synergy promotes endoderm-specific expression of ref-1 in C. elegans. Development 134, 445968 (2007).

123. Barbarulo, A. et al. Notch3 and canonical NF-kappaB signaling pathways cooperatively regulate Foxp3 transcription. J Immunol 186, 6199-206 (2011).

124. Terriente-Felix, A. et al. Notch cooperates with Lozenge/Runx to lock haemocytes into a differentiation programme. Development 140, 926-37 (2013).

125. Geimer Le Lay, A.S. et al. The tumor suppressor Ikaros shapes the repertoire of notch target genes in T cells. Sci Signal 7, ra28 (2014).

126. Kleinmann, E., Geimer Le Lay, A.S., Sellars, M., Kastner, P. \& Chan, S. Ikaros represses the transcriptional response to Notch signaling in T-cell development. Mol Cell Biol 28, 7465-75 (2008).

127. Witkowski, M.T. et al. Activated Notch counteracts Ikaros tumor suppression in mouse and human T-cell acute lymphoblastic leukemia. Leukemia 29, 1301-11 (2015).

128. Kim, H.S., Jeong, H., Lim, S.O. \& Jung, G. Snail inhibits Notch1 intracellular domain mediated transcriptional activation via competing with MAML1.

Biochem Biophys Res Commun 433, 6-10 (2013).

129. Gao, J. et al. RUNX3 directly interacts with intracellular domain of Notch1 and suppresses Notch signaling in hepatocellular carcinoma cells. Exp Cell Res 316, 149-57 (2010).

130. Sakano, D. et al. BCL6 canalizes Notch-dependent transcription, excluding Mastermind-like 1 from selected target genes during left-right patterning. Dev Cell 18, 450-62 (2010).

131. Tiberi, L. et al. BCL6 controls neurogenesis through Sirt1-dependent epigenetic repression of selective Notch targets. Nat Neurosci 15, 1627-35 (2012).

132. Dai, Q. et al. Common and distinct DNA-binding and regulatory activities of the BEN-solo transcription factor family. Genes Dev 29, 48-62 (2013).

133. Schwanbeck, $R$. The role of epigenetic mechanisms in Notch signaling during development. J Cell Physiol 230, 969-81 (2015). 
134. Endo, K. et al. Chromatin modification of Notch targets in olfactory receptor neuron diversification. Nat Neurosci 15, 224-33 (2011).

135. Farnsworth, D.R., Bayraktar, O.A. \& Doe, C.Q. Aging Neural Progenitors Lose Competence to Respond to Mitogenic Notch Signaling. Curr Biol 25, 3058-68 (2015). Example illustrating how presence of specific transcription factors can change a cell's response to Notch.

136. Felician, G. et al. Epigenetic modification at Notch responsive promoters blunts efficacy of inducing notch pathway reactivation after myocardial infarction. Circ Res 115, 636-49 (2014).

137. Martinez, A.M. et al. Polyhomeotic has a tumor suppressor activity mediated by repression of Notch signaling. Nat Genet 41, 1076-82 (2009).

138. Blokzijl, A. et al. Cross-talk between the Notch and TGF-beta signaling pathways mediated by interaction of the Notch intracellular domain with Smad3. J Cell Biol 163, 723-8 (2003).

139. Manderfield, L.J. et al. Hippo signaling is required for Notch-dependent smooth muscle differentiation of neural crest. Development 142, 2962-71 (2015).

140. O'Neil, J. et al. FBW7 mutations in leukemic cells mediate NOTCH pathway activation and resistance to gamma-secretase inhibitors. J Exp Med 204, 1813-24 (2007).

141. Oberg, C. et al. The Notch intracellular domain is ubiquitinated and negatively regulated by the mammalian Sel-10 homolog. J Biol Chem 276, 35847-53 (2001).

142. Fryer, C.J., White, J.B. \& Jones, K.A. Mastermind recruits CycC:CDK8 to phosphorylate the Notch ICD and coordinate activation with turnover. Mol Cell 16, 509-20 (2004).

143. Kourtis, N., Strikoudis, A. \& Aifantis, I. Emerging roles for the FBXW7 ubiquitin ligase in leukemia and beyond. Curr Opin Cell Biol 37, 28-34 (2015).

144. Davis, M.A. et al. The SCF-Fbw7 ubiquitin ligase degrades MED13 and MED13L and regulates CDK8 module association with Mediator. Genes Dev 27, 151-6 (2013).

145. Hoeck, J.D. et al. Fbw7 controls neural stem cell differentiation and progenitor apoptosis via Notch and C-Jun. Nat Neurosci 13, 1365-72 (2010).

146. Tetzlaff, M.T. et al. Defective cardiovascular development and elevated cyclin $\mathrm{E}$ and Notch proteins in mice lacking the Fbw7 F-box protein. Proc Natl Acad Sci U S A 101, 3338-45 (2004).

147. Guarani, V. et al. Acetylation-dependent regulation of endothelial Notch signalling by the SIRT1 deacetylase. Nature 473, 234-8 (2011).

148. Hein, K. et al. Site-specific methylation of Notch1 controls the amplitude and duration of the Notch1 response. Sci Signal 8, ra30 (2015).

149. Zheng, X. et al. Interaction with factor inhibiting HIF-1 defines an additional mode of cross-coupling between the Notch and hypoxia signaling pathways. Proc Natl Acad Sci U S A 105, 3368-73 (2008).

150. Espinosa, L., Ingles-Esteve, J., Aguilera, C. \& Bigas, A. Phosphorylation by glycogen synthase kinase-3 beta down-regulates Notch activity, a link for Notch and Wnt pathways. J Biol Chem 278, $32227-35$ (2003).

151. Song, J., Park, S., Kim, M. \& Shin, I. Down-regulation of Notch-dependent transcription by Akt in vitro. FEBS Lett 582, 1693-9 (2008).

152. Mund, T. et al. Disinhibition of the HECT E3 ubiquitin ligase WWP2 by polymerized Dishevelled. Open Biol 5 (2015).

153. Jung, J.G. et al. Notch3 interactome analysis identified WWP2 as a negative regulator of Notch3 signaling in ovarian cancer. PLoS Genet 10, e1004751 (2014). 
154. Ishitani, T. et al. Nemo-like kinase suppresses Notch signalling by interfering with formation of the Notch active transcriptional complex. Nat Cell Biol 12, 278-85 (2010).

155. Fernandez-Martinez, J. et al. Attenuation of Notch signalling by the Downsyndrome-associated kinase DYRK1A. J Cell Sci 122, 1574-83 (2009).

156. Borggrefe, T. et al. The Notch intracellular domain integrates signals from Wnt, Hedgehog, TGFbeta/BMP and hypoxia pathways. Biochim Biophys Acta 1863, 303-13 (2016).

157. Yatim, A. et al. NOTCH1 nuclear interactome reveals key regulators of its transcriptional activity and oncogenic function. Mol Cell 48, 445-58 (2012).

158. Fischer, A. \& Gessler, M. Delta-Notch--and then? Protein interactions and proposed modes of repression by Hes and Hey bHLH factors. Nucleic Acids Res 35, 4583-96 (2007).

159. Eddison, M., Le Roux, I. \& Lewis, J. Notch signaling in the development of the inner ear: lessons from Drosophila. Proc Natl Acad Sci U S A 97, 11692-9 (2000).

160. Djiane, A. et al. Dissecting the mechanisms of Notch induced hyperplasia. Embo J 32, 60-71 (2013).

161. Palomero, T. et al. Mutational loss of PTEN induces resistance to NOTCH1 inhibition in T-cell leukemia. Nat Med 13, 1203-10 (2007). Uncovered an indirect regulation of PTEN by HES genes in T-ALL

162. Serra, H. et al. PTEN mediates Notch-dependent stalk cell arrest in angiogenesis. Nat Commun 6, 7935 (2015). Contrast with 160, shows that PTEN is positively regulated by Notch activation in stalk cells.

163. Sundaram, M.V. The love-hate relationship between Ras and Notch. Genes Dev 19, 1825-39 (2005).

164. Flores, G.V. et al. Combinatorial signaling in the specification of unique cell fates. Cell 103, 75-85 (2000). Nice example showing how signals can be integrated through a common enhancer.

165. Doroquez, D.B. \& Rebay, I. Signal integration during development: mechanisms of EGFR and Notch pathway function and cross-talk. Crit Rev Biochem Mol Biol 41, 339-85 (2006).

166. Yoo, A.S., Bais, C. \& Greenwald, I. Crosstalk between the EGFR and LIN$12 /$ Notch pathways in C. elegans vulval development. Science 303, 663-6 (2004).

167. Lien, W.H. \& Fuchs, E. Wnt some lose some: transcriptional governance of stem cells by Wnt/beta-catenin signaling. Genes Dev 28, 1517-32.

168. Cordle, J. et al. A conserved face of the Jagged/Serrate DSL domain is involved in Notch trans-activation and cis-inhibition. Nat Struct Mol Biol 15, 849-57 (2008).

169. Chillakuri, C.R. et al. Structural analysis uncovers lipid-binding properties of Notch ligands. Cell Rep 5, 861-7 (2013).

170. van Tetering, G. et al. Metalloprotease ADAM10 is required for Notch1 site 2 cleavage. J Biol Chem 284, 31018-27 (2009).

171. Kovall, R.A. \& Blacklow, S.C. Mechanistic insights into Notch receptor signaling from structural and biochemical studies. Curr Top Dev Biol 92, 3171 (2010).

172. Nam, Y., Sliz, P., Song, L., Aster, J.C. \& Blacklow, S.C. Structural basis for cooperativity in recruitment of MAML coactivators to Notch transcription complexes. Cell 124, 973-83 (2006). Together with 172, made a key contribution by revealing how NICD binds to CSL and forms an interface that recruits Mam. Led to the design of inhibitors.

173. Wilson, J.J. \& Kovall, R.A. Crystal structure of the CSL-NotchMastermind ternary complex bound to DNA. Cell 124, 985-96 (2006). 
174. Lin, S. et al. DDX5 is a positive regulator of oncogenic NOTCH1 signaling in T cell acute lymphoblastic leukemia. Oncogene 32, 4845-53 (2013).

175. Jung, C., Mittler, G., Oswald, F. \& Borggrefe, T. RNA helicase Ddx5 and the noncoding RNA SRA act as coactivators in the Notch signaling pathway. Biochim Biophys Acta 1833, 1180-9 (2013).

176. Wang, H., Zang, C., Liu, X.S. \& Aster, J.C. The role of Notch receptors in transcriptional regulation. J Cell Physiol 230, 982-8 (2015).

177. Lee, M.C. \& Spradling, A.C. The progenitor state is maintained by lysinespecific demethylase 1-mediated epigenetic plasticity during Drosophila follicle cell development. Genes Dev 28, 2739-49 (2014).

178. Liefke, R. et al. Histone demethylase KDM5A is an integral part of the core Notch-RBP-J repressor complex. Genes Dev 24, 590-601 (2010).

179. Maier, D. Hairless: the ignored antagonist of the Notch signalling pathway. Hereditas 143, 212-21 (2006).

180. Rayon, T. et al. Notch and hippo converge on Cdx2 to specify the trophectoderm lineage in the mouse blastocyst. Dev Cell 30, 410-22 (2014).

181. Sacilotto, N. et al. Analysis of DIl4 regulation reveals a combinatorial role for Sox and Notch in arterial development. Proc Natl Acad Sci U S A 110, 118938 (2013).

182. Lopez-Arribillaga, E. et al. Bmi1 regulates murine intestinal stem cell proliferation and self-renewal downstream of Notch. Development 142, 41-50 (2015). 


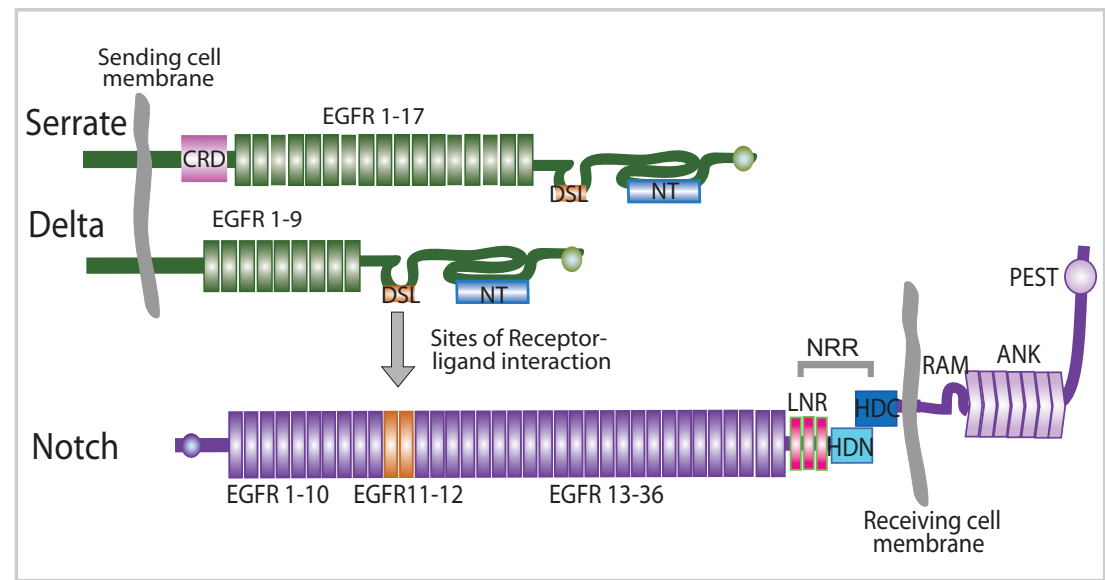


Bray_Figure 1
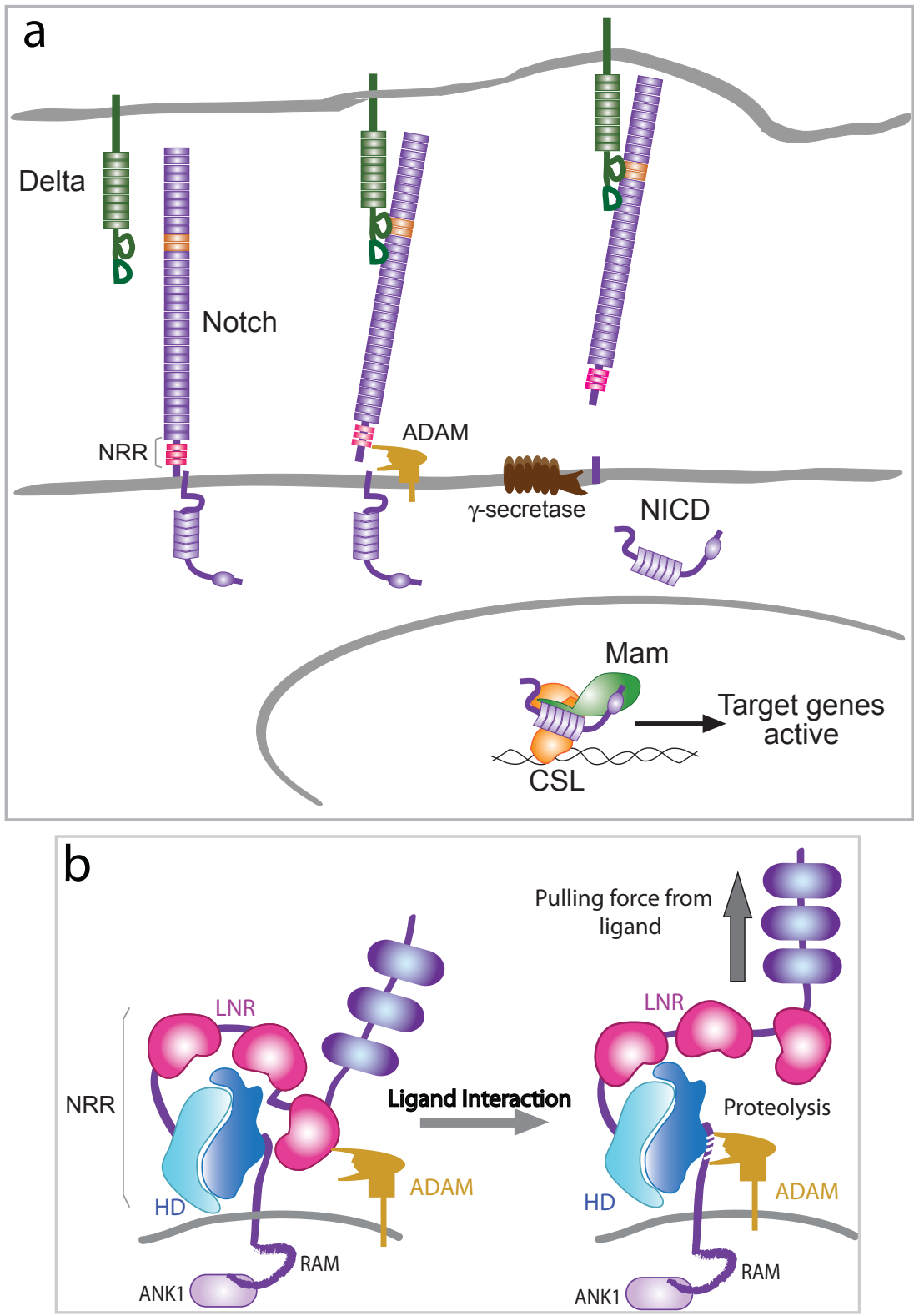
a
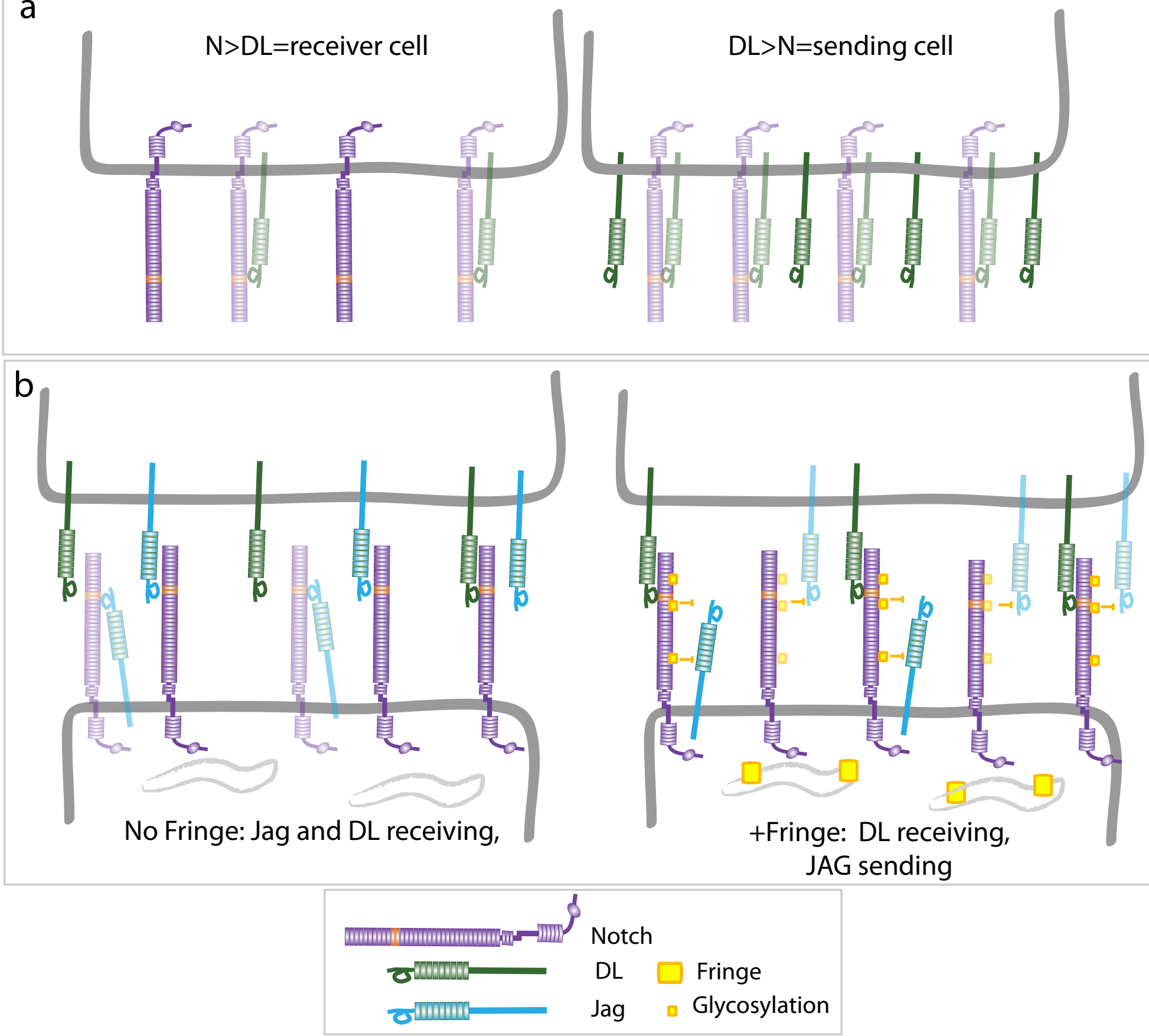


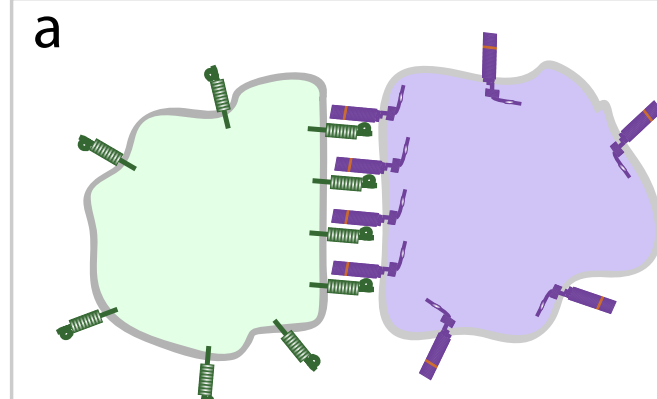

Large surface

Notch response 1
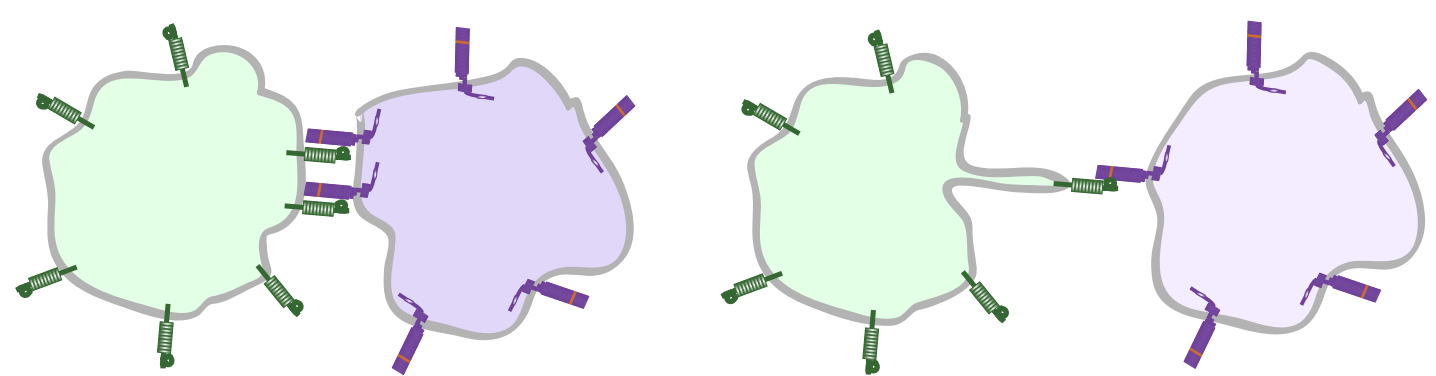

Small surface

Notch response 2

filopodia

Notch response 3

b

Notch defective
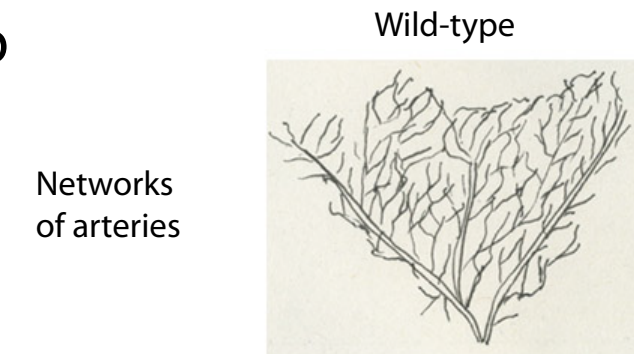

Spaced

Sensory

Organs
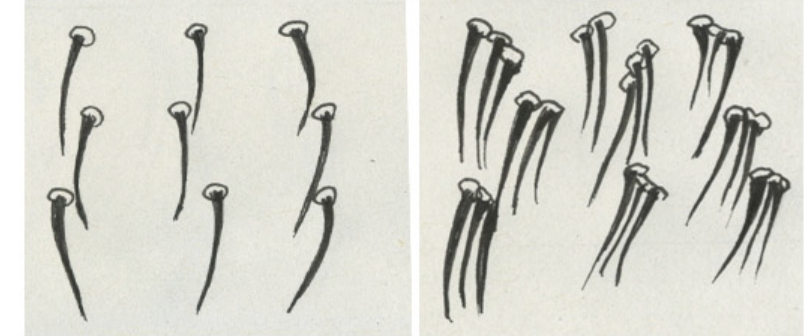

Pigmented

Melanophore

stripes
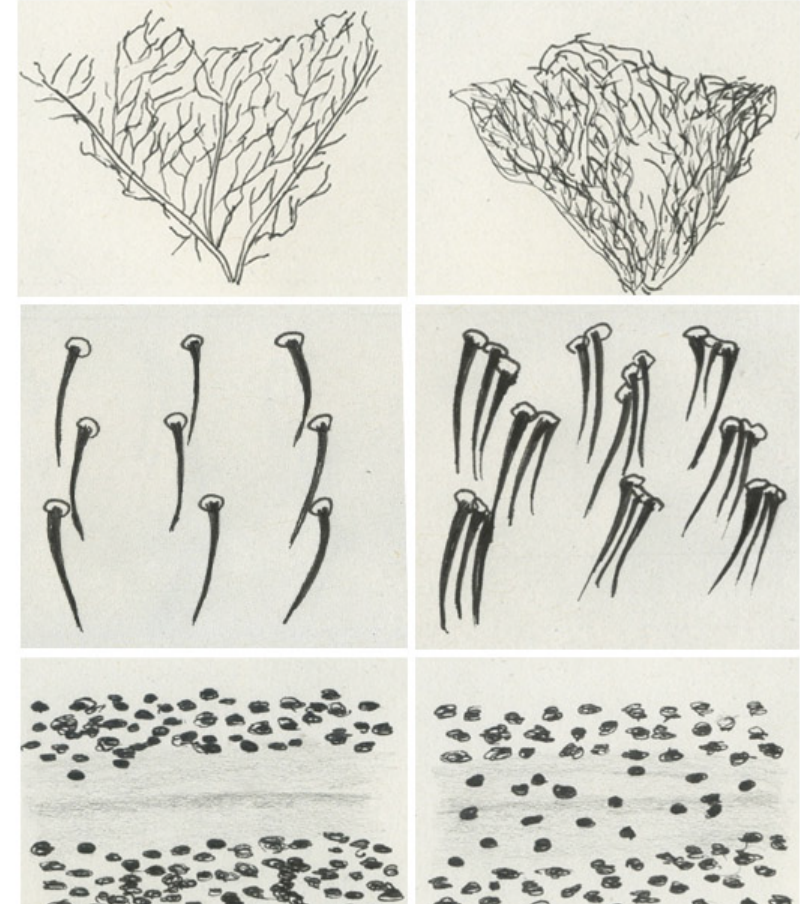
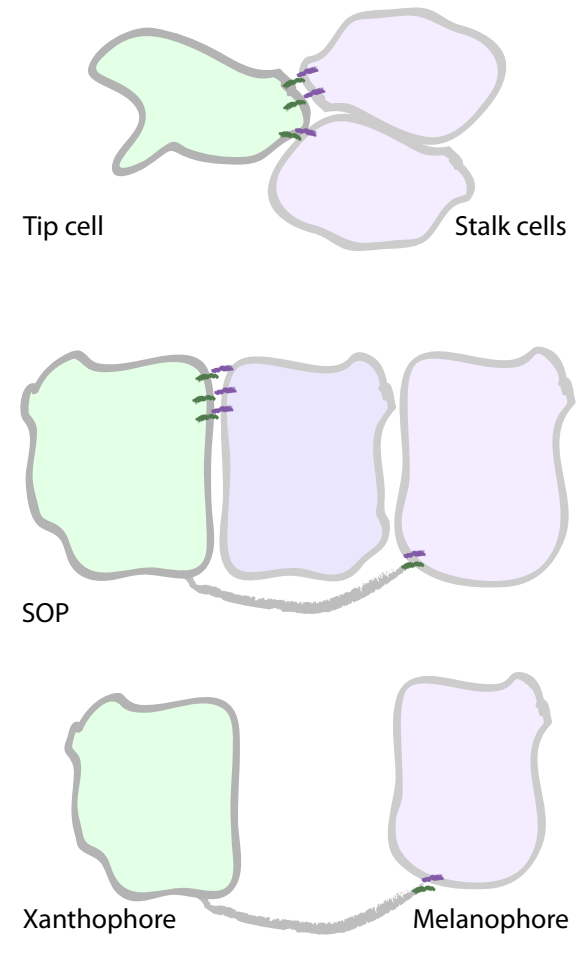
Bray_Figure 4

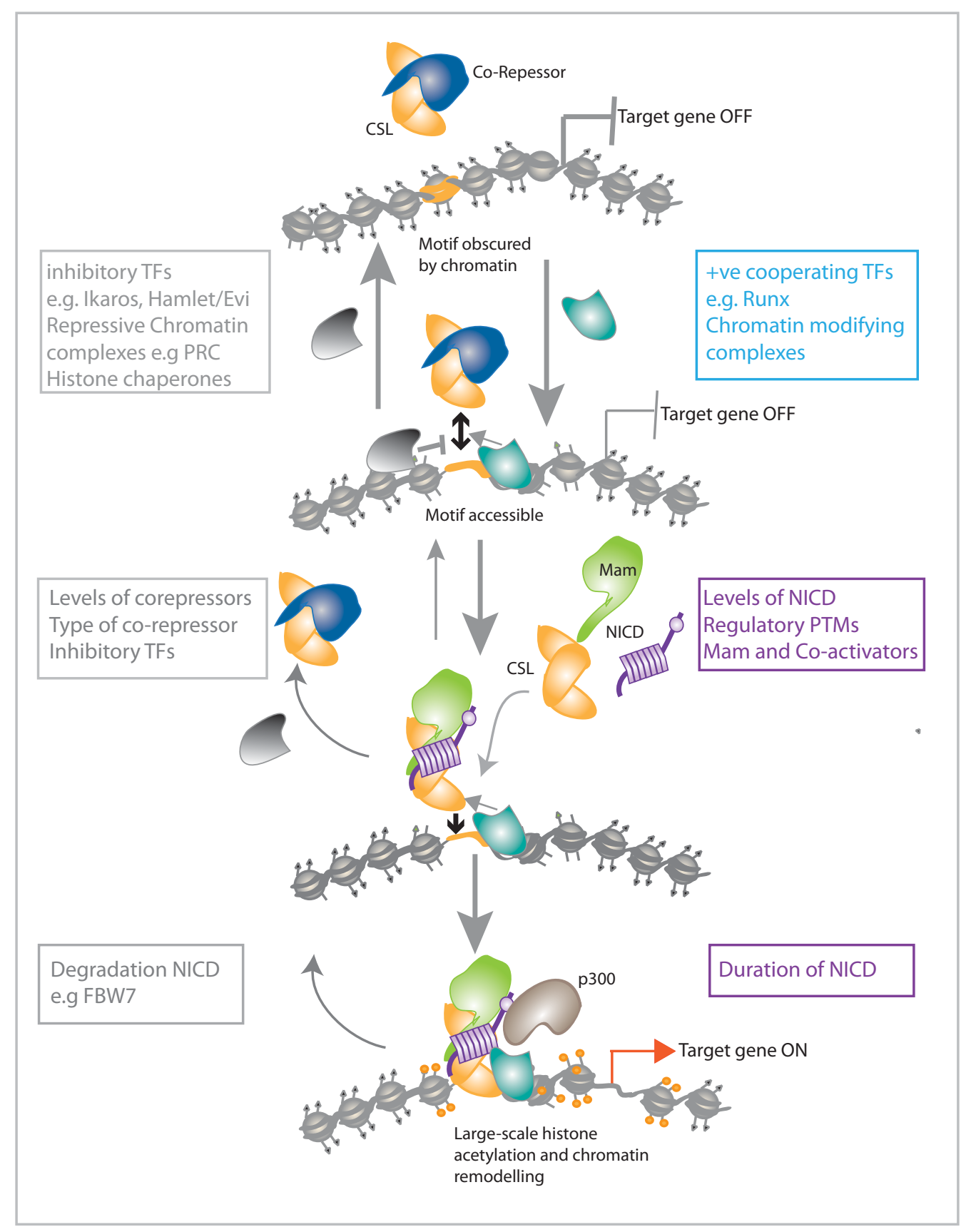


Bray_Figure 5

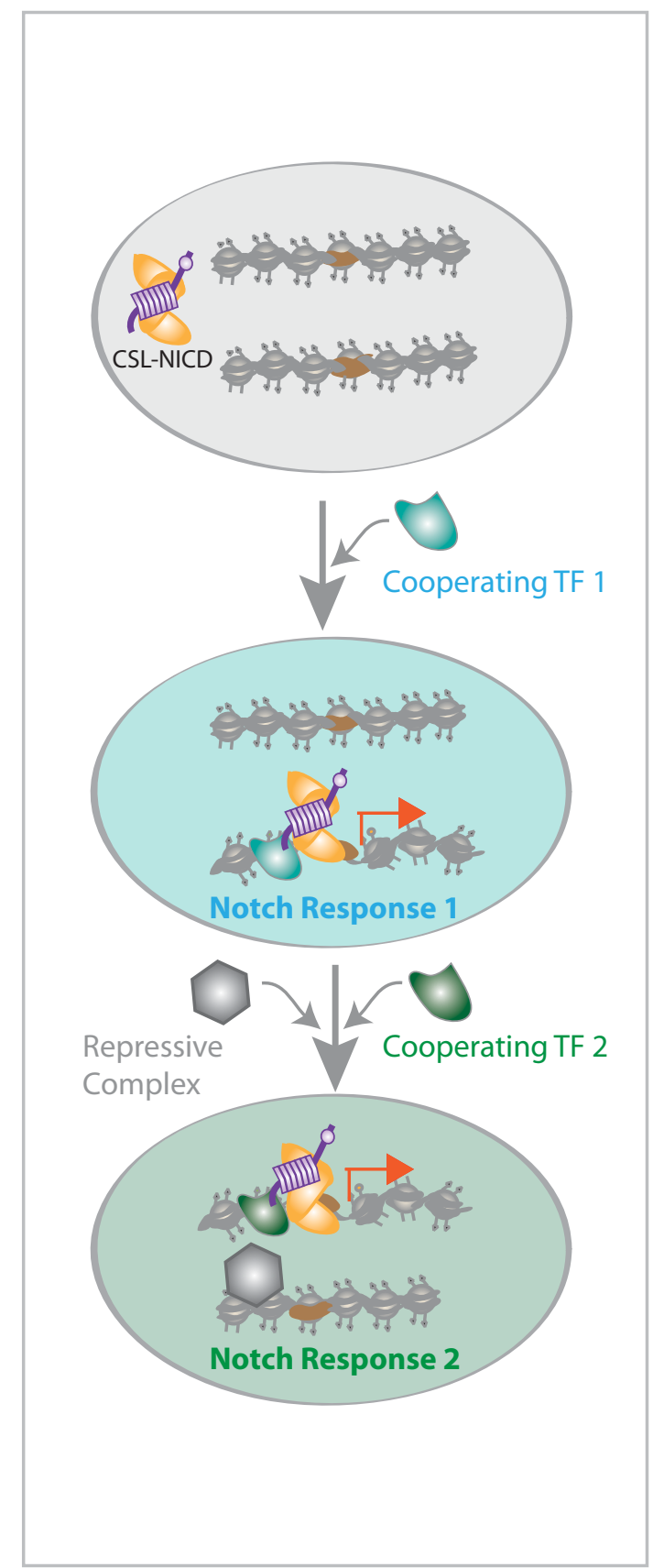

